\title{
Multimodal parcellations and extensive behavioral profiling tackling the hippocampus gradient
}

Anna Plachti ${ }^{1,2}$, Simon B. Eickhoff ${ }^{1,2}$, Felix Hoffstaedter ${ }^{1,2}$, Kaustubh R. Patil $^{1,2}$, Angela R. Laird ${ }^{4}$, Peter T. Fox ${ }^{5}$, Katrin Amunts ${ }^{2,3}$ \& Sarah Genon ${ }^{1,2,6}$

1 Institute of Systems Neuroscience, Heinrich Heine University Düsseldorf, Düsseldorf, Germany;

2 Institute of Neuroscience and Medicine (INM-1, INM-7), Research Centre Jülich, Jülich, Germany;

3 C. \& O. Vogt Institute for Brain Research, Heinrich Heine University, Düsseldorf. Germany;

4 Department of Physics, Florida International University, Miami, FL, USA;

5 Research Imaging Institute, University of Texas Health Science Center at San Antonio, TX, USA;

6 GIGA-CRC In vivo Imaging, University of Liege, Liege, Belgium.

contact: Telefon: $+49246161-1409$

Fax: +49 2461 61-1880

E-Mail: a.plachti@fz-juelich.de 
Abstract: The hippocampus displays a complex organization and function that is perturbed in many neuropathologies. Histological work revealed a complex arrangement of subfields along the medial-lateral and the ventral-dorsal dimension, which contrasts with the anterior-posterior functional differentiation. The variety of maps has raised the need for an integrative multimodal view. We applied connectivitybased parcellation to 1) intrinsic connectivity 2) task-based connectivity and 3) structural covariance, as complementary windows into structural and functional differentiation of the hippocampus. Strikingly, while functional properties (i.e., intrinsic and task-based) revealed similar partitions dominated by an anterior-posterior organization, structural covariance exhibited a hybrid pattern reflecting both functional and cytoarchitectonic subdivision. Capitalizing on the consistency of functional parcellations, we defined robust functional maps at different levels of partitions, which are openly available for the scientific community. Our functional maps demonstrated a head-body and tail partition, subdivided along the anterior-posterior and medial-lateral axis. Behavioral profiling of these fine partitions based on activation data indicated an emotion-cognition gradient along the anterior-posterior axis and additionally suggested a self-world centric gradient supporting the role of the hippocampus in the construction of abstract representations for spatial navigation and episodic memory.

Keywords: anterior-posterior, gradient, medial temporal lobe, structural covariance, map. 


\section{Introduction}

The hippocampus is involved in a variety of tasks ranging from memory, learning, navigation and emotion (Fanselow and Dong 2010; Moser and Moser 1998; Poppenk et al. 2013; Prince et al. 2005; Strange et al. 2014). However, an integrative conceptual framework is currently lacking to account for this diversity of behavioral findings. To progress in that direction, first, a better understanding of the hippocampus' organization and function is crucially needed to shed light on its role in a range of behavioral aspects and second, a common generic map would be highly useful to further support crossstudies comparison and integration.

As far, two opposing organizational patterns were introduced in the past. The first mapping based on cytoarchitecture has evidenced a subdivision into subfields (CA1-4, dentate gyrus, subiculum) along the medial-lateral and ventro-dorsal axes as illustrated in Figure 1, Amunts et al. 2005). In parallel to this organization, an organization into subregions (head, body, tail) along the anterior-posterior (longitudinal) axis (Moser \& Moser 1998; Lepage et al. 1998; Fanselow \& Dong 2010; Poppenk et al. 2013; Strange, et al. 2014) commonly emerged across a variety of in-vivo approaches such as electrophysiology (Komorowski et al. 2013) and connectivity-based parcellation (CBP) (see Figure 1,(Amunts et al. 2005; Chase et al. 2015; Robinson et al. 2015)).

In line with the histological work and despite evidence of functional anterior-posterior differences, many in-vivo and ex-vivo studies in the human hippocampus used a-priori segmentation into subfields based on either an automated or a manually delineation in the anatomical MRI scans (see Figure 1, (Adler et al. 2014; Adler et al. 2018; de Flores et al. 2015). Such segmentation into subfields has the advantage of using the histological "ground truth" as an a-priori representation, but has the disadvantage of neglecting 
higher order features, such as the rich long-range connectivity of the hippocampus, which contributes to its functional organization. Thus, the knowledge from this onesided perspective should be complemented by a CBP approach, which now allows the combination of different MRI measurements within the whole hippocampus hence potentially probing different aspects of its organization.

CBP is an in-vivo brain-mapping method that characterizes the organization of the brain based on connectivity estimates, usually derived from MRI (Eickhoff in press). CBP can be applied on any estimates of connectivity from MRI data (functional or structural) with different types of connectivity measurements being usually referred to as different CBP-modalities. Across the previous years, evidence have been brought that CBP can capture organizational aspects that were previously revealed by tracing studies, as well as by histological work (Behrens et al. 2003; Lambert et al. 2017). Additionally, CBP was shown to be sensitive to functional distinction, for example, replicating the supplementary motor area (SMA) and pre-SMA differentiation evidenced by functional signal during task (Johansen-Berg et al. 2004). Hence this approach appears to identify regional differentiation supported by local microarchitecture, connectivity and local functional signal to some extent.

In the present study, we focused on the functional connectivity between hippocampus' voxels and all grey matter voxels. In other words, we examined long-range (whole brain) connectivity by computing for every hippocampal voxel its individual connectivity fingerprint with all other grey matter voxels. Based on the (dis-)similarity of connectivity fingerprints the voxels were clustered into either same or different partitions. CBP has the advantage to be model-free and unsupervised hence offering maps that optimally represent the data at hand. It has already been used in previous studies to examine hippocampal organization. Nevertheless, previous work focused 
mainly on a single CBP modality, either structural connectivity (Adnan et al. 2016) or meta-analytic connectivity modeling (MACM) (Chase et al. 2015; Robinson et al. 2015; Robinson et al. 2016). Examining structural connectivity, Adnan et al. (2016) proposed a bipartite anterior-posterior subdivision of the hippocampus, which contrasted with MACM parcellations revealing a more detailed architecture. This latter modality yielded a three-part organization for the left hippocampus and a 5-partite structure for the right hippocampus along the anterior-posterior axis (Robinson et al. 2015; Robinson et al. 2016), while the subiculum subfield was subdivided into five modules (Chase et al. 2015). In sum, uni-modal CBP has thus far provided evidence for an anterior-posterior organization of the hippocampus, but at different levels of partition across studies and even across hemispheres. This variety of partition schemes hinders a deep investigation of the functional relevance of the anterior-posterior differentiation, and also complicates the study of hippocampus dysfunction in brain pathology.

In this latter perspective, a common set of maps of the hippocampus for MRI investigation would be highly useful. Across the previous years, one major avenue of neuroimaging research of brain pathology has developed from phenotype (such as cognitive performance or symptoms) prediction approaches based on multivariate pattern analyses applied to large-scale clinical datasets (Zhang et al. 2016). In this promising avenue of research, individual voxels have to be compressed into homogeneous subregions in which the measurements (e.g. fMRI signal) can be summarized. This compression is most of the time required not only for computational purposes, but also for post-hoc investigations of subregions contributing to the predictions. In this framework, the compression should be based on robustly defined maps that would represent a universal framework for comparison and integration of results across studies. 
In this study, we investigated hippocampal functional organization using a multimodal CBP approach to generate robust functional hippocampal maps based on hippocampus whole-brain connectivity profiles (Eickhoff et al. 2015; Eickhoff in press). To do so, we focused on two purely functional modalities: MACM-CBP and resting-state functional connectivity (RSFC-CBP). Despite showing convergence (Reid et al. 2016) and being conceptually related, these two modalities are based on very different types of data and methods. MACM reflects functional organization during task and is computed from whole-brain co-activation peaks in activation databases such as BrainMap. For each hippocampal voxel we obtained a whole-brain co-activation profile. RSFC, on the other hand, reflects the functional connectivity estimated in the unconstrained function of the brain and is computed at the subject-level. For each individual hippocampal voxel we obtained its functional connectivity profile to all the other grey matter voxels in the brain. RSFC is based on resting state functional MRI (RS-fMRI), which is known to be prone to noise due to various artifacts (Power et al. 2015; Satterthwaite et al. 2017; Satterthwaite et al. 2013; Van Dijk et al. 2012), but the choice of an optimal denoising strategy has remained relatively unexplored in the particular framework of CBP. For that reason, as a preliminary step in the present study, we performed a systematic evaluation of different denoising methods in order to favor stable partitions with high biological validity for RSFC-CBP. We aimed to generate a functional subdivision of the hippocampus that would be stable across subjects and CBP modalities offering a representation that would be optimal for any type of functional signal (such as taskbased fMRI activations, RS-fMRI or PET).

Nevertheless, to complement this purely functional parcellation and to start building a scientific bridge with previous structural mapping modalities, we additionally examined the subdivision of the hippocampus based on structural covariance (SC-CBP), which 
represents on group-level the co-variation of hippocampal voxels with all the other brain voxels. SC stands at an ambiguous place in the mapping approaches. On one hand, it is assumed to reflect functional dependencies between regions through synchronous firing of neurons reflecting functional neuroplasticity (Alexander-Bloch et al. 2013; Evans 2013). Accordingly, SC and RSFC are conceptually related to each other (Kotkowski et al. 2018), as indicated by structural changes through function (Seeley et al. 2009) although both are technically two distinct modalities. However, on the other hand, SC is based on structural changes (Alexander-Bloch et al. 2013; Mechelli et al. 2005) and thus should be influenced by the underlying structural organization like gene expression during neurodevelopment and direct structural connectivity through monosynaptic connection as indicated in a recent rodent study (Yee et al. 2017). In sum, SC is assumed to reflect common influences of certain factors on microstructure be it synaptogenesis based on functional synchronous firing, connectivity as direct monosynaptic connection, or gene expression in synapses development. Therefore, we expected that SC-CBP would to some extent confirm functional organization, and additionally, provide anatomical information conveyed in brain structure to complement our understanding of the hippocampal functional topography and provide an alternative map for studies capitalizing on structural MRI data. 
Ex-vivo mapping: from
histology to probabilistic

maps
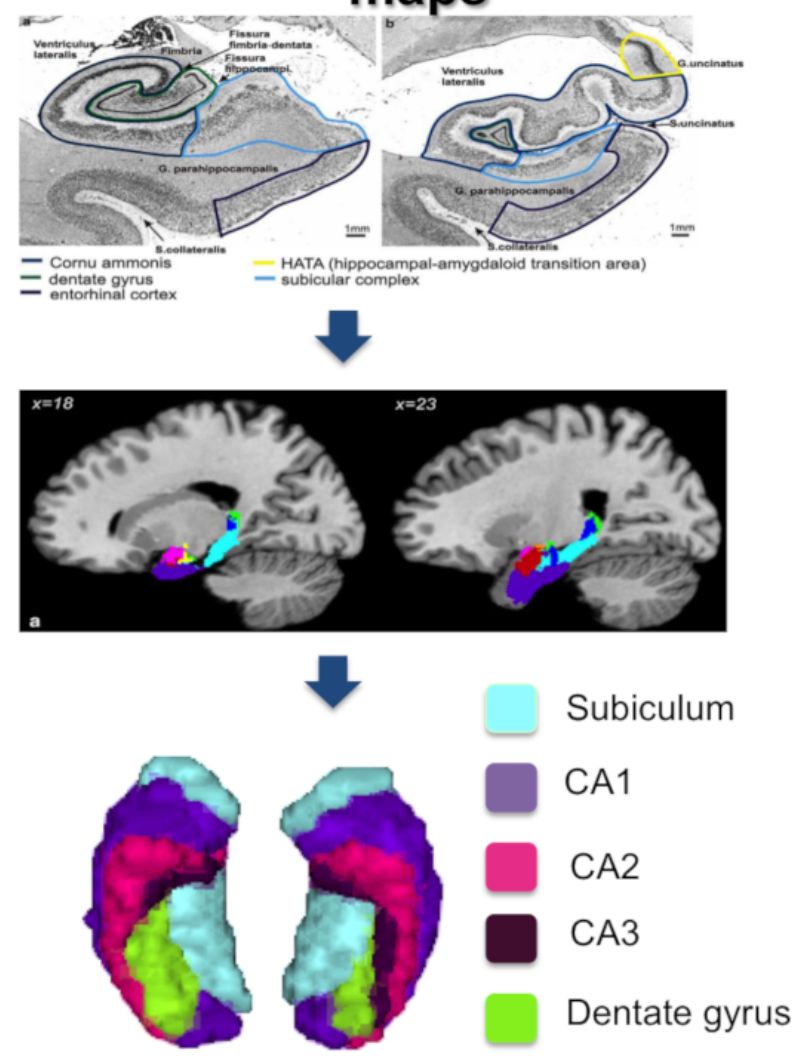

Amunts et al. 2005

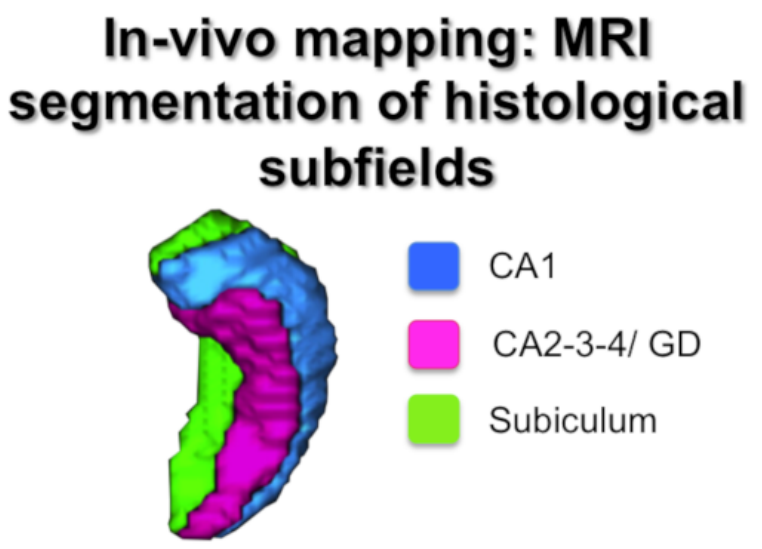

Chetelat et al. (2008)

\section{In-vivo mapping: MACM- CBP}

Anterior
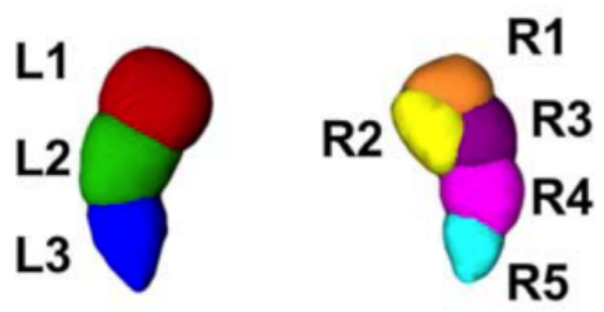

\section{Posterior}

Robinson et al. 2015

Figure 1. Hippocampal mapping based on histology, structural MRI segmentation, and CBP method. (Images reproduced with permission from publishers.)

Our final objective was to characterize the obtained cross-modal functional maps in terms of associated behavioral functions via a quantitative approach of activation studies (e.g., using the BrainMap or NeuroSynth databases). Importantly, our conceptual objective here was not to identify specific behavioral functions segregated into different subregions of the hippocampus, but rather to assess the functional relevance and integration of the organization pattern in terms of cognitive information processing. Several hypotheses have been proposed in the past to describe the anterior-posterior 
differentiation in terms of psychological functions. But these hypotheses usually pertain to a specific psychological or neuroscientific research domain and hence could not account for pluripotency of the engagement of the hippocampus across psychological domains. As far, two main hypotheses derived from psychological ontologies have been proposed in that regard: an emotional-cognitive dimension (Moser and Moser 1998) and an encoding-retrieval dimension (Kim 2015; Lepage et al. 1998; Prince et al. 2005). Further investigations have proposed a novelty-familiarity (Strange et al. 1999) and an imagination-perception differentiation along the anterior-posterior axis (Zeidman and Maguire 2016). However, a common framework accounting for the relevance of the organization of the hippocampus across domains of human behavior is still lacking. The current study aimed to fill this gap by performing behavioral profiling (Genon et al. 2018b) of hippocampus subregions using thousands of activation studies collected across two different databases using different behavioral taxonomies. Such a quantitative approach offers an overview, which can be used as a starting point to build an integrative theory.

Thus, the objectives of the present study were two-fold 1) a conceptual objective of understanding hippocampal organization as revealed across different neurobiological properties and its relevance in terms of cognitive information processing, and 2) a mapping objective to provide robust and fine-grained partitions of the hippocampus. While current high-level parcellations (Glasser et al. 2016; Schaefer et al. 2017) have focused on the cerebral cortex they neglected crucial subcortical structures. A consensual robust map of the bilateral hippocampus is still missing which in turn can help to study its structure and function across the lifespan as well as in disease. Our study was designed to offer such partitions and their patterns of associations with 
behavioral functions. These resources are openly available to the scientific community via ANIMA database (http://anima.fz-juelich.de/).

\section{Materials and Methods}

The bilateral hippocampi were parcellated using different connectivity modalities. Taskbased connectivity was examined with MACM performed on reported activation peaks across paradigms in the BrainMap database. RSFC-CBP was performed at the subject level for a sample of participants from the Human Connectome Project (HCP) while SCCBP was performed at the group level using the structural MRI data of the same HCP sample (Smith et al. 2013; Ugurbil et al. 2013). The main methodological differences between these two CBP modalities are illustrated in Supplemental material methods I.5. After computing parcellations for each modality, we established a functional map of the hippocampus by merging the functional parcellations (i.e. RSFC and MACM, that showed the highest convergence) into one hippocampal map, hence representing a cross-modal consensus map. Finally, we characterized our cross-modal consensus map at high granularity with regards to behavioral functions using BrainMap and NeuroSynth databases.

\subsection{Volume of interest}

We defined our VOI as a consortium of the cytoarchitectonic maps, available in the SPM Anatomy Toolbox 2.0 (Eickhoff et al. 2005), and the macro anatomically-defined Harvard-Oxford Structural Probability Atlas (http://neuro.imm.dtu.dk/wiki/HarvardOxford_Atlas) (Desikan et al. 2006). The hippocampal formation included the following 
subfields: CA1-3, dentate gyrus and subiculum. The total number of voxels in a $2 \mathrm{~mm} \times 2$ $\mathrm{mm} \times 2 \mathrm{~mm}$ space in the right hippocampus was $865\left(6920 \mathrm{~mm}^{3}\right)$ and that of the left hippocampus was $831\left(6648 \mathrm{~mm}^{3}\right)$ voxels.

\subsection{Sample}

The sample was obtained from the longitudinal study of the Human Connectome Project (Van Essen and Ugurbil 2012) representing one of the best openly accessible MRI datasets. We included unrelated participants in order to avoid heritability effects. The sample consisted of $n=323$ young adults (age: $22-37$ years, mean age: 28.2 years, $50.7 \%$ females). All participants gave their written statement of agreement, and the analyses of the data were approved by the ethical committee of the Heinrich Heine University Düsseldorf.

\subsection{MRI measurements}

Structural MRI. All scans were acquired on a 3T MRI scanner of Siemens Skyra (Siemens AG, Erlangen, Germany) with a 32-channel coil (Van Essen and Ugurbil 2012). The 3D structural T1-weighted MRI scans were performed with a MPRAGE sequence (256 sagittal slices in a single slab, $T R=2400 \mathrm{~ms}, T E=2.14 \mathrm{~ms}, \mathrm{TI}=1000 \mathrm{~ms}, \mathrm{FoV}=224 \times 224$ $\mathrm{mm}$, flip angle $=8 \circ$, voxel size $=0.7 \times 0.7 \times 0.7 \mathrm{~mm}^{3}$ ). Preprocessing of the MRI data was performed with SPM8 (Statistical Parametric Mapping) and the VBM8 toolbox, running on Matlab R2014a. Structural images were normalized with the DARTEL algorithm to the ICBM-152 template using both affine and non-linear spatial normalization. Images were bias-field corrected and segmented into grey matter, white matter, and 
cerebrospinal fluid tissues. The grey matter segments were then modulated for nonlinear transformations only and subsequently smoothed with an isotropic Gaussian kernel (full-width-half-maximum = 8).

Resting-state functional MRI. The acquisition of resting state fMRI with opposite phase encoding directions (L/R and R/L) was performed with an EPI sequence for a duration of 30 min (eyes open and fixated on a hair cross), 72 slices covering the whole brain (TR $=720 \mathrm{~ms}$, using a multiband factor of $8, T E=33 \mathrm{~ms}, \mathrm{FoV}=208 \times 180 \mathrm{~mm}$, flip angle $=$ 52॰, voxel resolution $=2 \times 2 \times 2 \mathrm{~mm}^{3}$ (Smith et al. 2013; Ugurbil et al. 2013). During preprocessing we corrected for movements by affine two-pass registration and aligned the images to the first volume and to the mean of the volumes. Variance explained by six motion parameters from the realignment and their first derivatives were regressed out. Spatial normalization to the Montreal Neurological Institute (MNI) was carried out for the average EPI scans for each subject using the unified segmentation approach (Ashburner and Friston 2005). We applied a band-pass filter with the cut-off frequencies of 0.01 and $0.08 \mathrm{~Hz}$. The images were smoothed with the isotropic Gaussian Kernel (fullwidth-half-maximum $=5$ ).

\subsection{Connectivity-based parcellation}

\subsubsection{Parcellation based on structural covariance (SC-CBP)}

For each subject, structural covariance was measured by computing the Pearson's correlation coefficient between grey matter volume values of the hippocampus' VOI voxels (seed voxels) and all other brain grey matter voxels across the whole sample. 
This procedure yielded a seed voxels by target voxels connectivity matrix at the group level that was then used for clustering (see Supplemental material I.5 Fig.4).

\subsubsection{Parcellation based on resting-state functional connectivity (RSFC-CBP)}

Resting state functional connectivity between two brain regions was estimated by computing Pearson's correlation between time series of blood oxygen level-dependent signal (BOLD) at the subject level (Biswal et al. 1995; Buckner et al. 2013). For each seed voxel in the VOI we calculated the correlation with every other grey matter voxel of the brain (see Supplemental material I.5 Fig.4). Correlation values were then standardized using the Fisher's Z-transformation.

\subsubsection{Temporal MRI preprocessing}

The goal of denoising is to remove or at least to reduce the contribution of any artifacts and confounds that contaminate neurally generated BOLD-signal. Noise in RSFC can result from scanner artifacts (Ojemann et al. 1997), subject movement (Power et al. 2014; Satterthwaite et al. 2013; Van Dijk et al. 2012) and physiological processes (Birn et al. 2006). Standard denoising approaches aim to regress out variance that is driven by noise in the measured BOLD signal. One simple approach to do so relies on the calculation of global signal or/and signal in two specific non-grey matter tissues (i.e., white matter and cerebrospinal fluid), which are assumed to reflect artifacts. An alternative approach capitalizes on machine-learning techniques (e.g., FIX) to automatically identify potential noise in the data. With these approaches, the part of variance related to noise is typically first estimated and then regressed out from time- 
series. Several variants have been developed over the previous years and we described below the most commonly used strategies.

\subsubsection{Global signal regression}

In global signal regression (GSR), the mean fMRI signal across all brain voxels is regressed out (Desjardins et al. 2001; Macey et al. 2004). The underlying axiom is that any fluctuations that are measured globally are not attributable to neural activity but have physiological or mechanical origin (Bianciardi et al. 2009; Birn et al. 2006; Caballero-Gaudes and Reynolds 2017; Wise et al. 2004). Although it is unclear what exactly is reflected in the global signal and to what extent signal or nuisance is regressed out, it is still widely used. In this context of scientific uncertainty, the consequences of GSR on RSFC should be considered.

\subsubsection{White matter-, cerebrospinal fluid signal regression}

Another alternative is to estimate nuisance regressors from white matter (WM) and cerebrospinal fluid signal (CSF) (Anderson et al. 2011; Hallquist et al. 2013; Jo et al. 2013; Jo et al. 2010; Power et al. 2012; Weissenbacher et al. 2009; Yan et al. 2013). The signal's fluctuations in these parts of the brain are assumed to reflect drifts mainly caused by cardiac and respiratory effects (Dagli et al. 1999; de Munck et al. 2008; Van Dijk et al. 2010; Windischberger et al. 2002). To measure the mean signal across these regions, we created subject-specific masks by co-registering the WM and CSF templates to each individuals' space and subsequently, regressed out the mean signal computed within these masks. Note that the subject-specific templates were eroded in order to 
remove voxels on the edge of the mask that relate to grey matter and do not contain pure WM/CSF tissue (Caballero-Gaudes and Reynolds 2017; Jo et al. 2010).

\subsubsection{FMRIB's ICA-based X-noiseifier}

FMRIB's ICA-based X-noiseifier (FIX) is based on a machine learning approach, in which RS-fMRI signal has been decomposed into components of neural and non-neural sources by applying an independent component analysis (ICA) method (Beckmann and Smith 2004; Cole et al. 2010; Salimi-Khorshidi et al. 2014). FIX classifies the ICA components into relevant signal and noise-related components (Cole et al. 2010; Salimi-Khorshidi et al. 2014). We used the default classifier trained on a standard fMRI dataset, which has been shown to achieve 95\% accuracy (Salimi-Khorshidi et al. 2014). For each participant spatial ICA was performed using FSL's MELODIC toolbox (Beckmann and Smith 2004) and subsequently noise-variance components were regressed out.

In this study, we evaluated the denoising techniques both individually or in combination as recently suggested by Burgess et al. (2016) (see Supplemental material I.4 methods). We investigated six different strategies:

(1) Standard motion regression with 24 regressors without additional explicit denoising, termed as "no denoising" emphasizing no additional transformations.

(2) Regression of the averaged fMRI signal across all voxels of the brain (GSR).

(3) Regression of white matter and cerebro-spinal fluid-related variance (WM/CSF).

(4) Neutralization of 'bad' components of fMRI signal decomposed by ICA (FIX).

(5) A combination of FIX and GSR regression (FIX+GSR).

(6) A combination of FIX and WM/CSF regression (FIX+WM/CSF). 


\subsubsection{Parcellation based on meta-analytic connectivity modeling (MACM-CBP)}

From a computational point of view, MACM substantially differs from the aforementioned approaches since connectivity is not computed from collected MRI data as done for RSFC and SC but meta-analytically across activation foci of neuroimaging studies and paradigms archived in the BrainMap database (Laird et al. 2011) (http://www.brainmap.org). All experiments in BrainMap that were associated with each seed voxel or in the immediate vicinity of activation were considered. To account for spatial uncertainty, a spatial filter was systematically varied by including the closest 20 to 200 experiments in steps of 5 (for more details, see (Clos et al. 2013; Clos et al. 2014; Genon et al. 2017; Genon et al. 2018c). For each seed voxel, a meta-analytical coactivation likelihood profile for every other brain voxel given each of the 25 filter sizes was computed (with revised ALE algorithm (Eickhoff et al. 2012). The final CBP analysis was performed in the filter range of 100 to 148 experiments for the right hippocampus and in the filter range of 82 to 130 for the left hippocampus. An optimal filter range was defined based on the consistency of each voxels' cluster assignment across all the filter sizes (see Clos et al. 2013; (Chase et al. 2015; Clos et al. 2013; Genon et al. 2017) (see in Supplementary Methods I.3).

\subsection{Clustering method}

In line with previous studies, we used k-means (using MATLAB software 2014a) clustering, which showed good agreement with spectral clustering and outperformed hierarchical clustering (Arslan et al. 2018). The repetition number was set to 500, which 
almost doubled the recommended number of 256 repetitions (Nanetti et al. 2009), and the iteration number was set to 255. We examined six levels of granularity (levels of partitions) ranging from $k=2$ to $k=7$ since previous work has reported stable cluster solutions at different level of partitions (2,3 and 5 (Adnan et al. 2016; Chase et al. 2015; Robinson et al. 2015; Robinson et al. 2016)). The clustering was performed at the subject-level for RSFC and at the experiments range (filter range) for MACM while it was performed at the group-level with average across bootstrap resampling for SC. Modalityspecific and group-specific parcellations were achieved by assigning the hippocampal voxels to its most frequent cluster's label (i.e. by using the mode) across subjects, filter sizes and bootstrapping samples.

\subsection{Measurement of stability and consistency of parcellations}

In this work, we estimated stability and consistency of the partitions yielded by RSFCCBP since this modality is particularly challenging in terms of its sensitivity to noise, inter-individual variability (Mueller et al. 2013) and its dynamic nature (Hutchison et al. 2013). We examined both criteria in this CBP framework, but emphasized consistency over stability, as we aimed for biological validity in addition to stability by capturing convergent organizational characteristics across CBP modalities. In line with previous study (Varikuti et al. 2017), we considered the possibility that high stability within RSFC could be influenced by 'structured noise', which when regressed out might result in apparently lower stability but preserving biological relevance or even enhancing it.

We used two procedures in order to cross-validate our findings: (1) split-half (LaConte et al. 2003; Strother et al. 2002) to estimate the stability within a CBP modality (i.e. 
RSFC) and (2) bootstrap resampling with replacement to assess consistency between CBP modalities (i.e. RFSC vs MACM) (Bellec et al. 2010; Efron 1979).

In contrast to previous studies, we here assumed that multiple partitions at different granularities might be valid representations of the hippocampus organization, but at different levels. Accordingly, we focused on split-half and bootstrap resampling instead of internal validity criteria such as the silhouette value or the percentage of misclassified voxels as these latter metrics probe optimal data representation within the specific modality at hand, while we here aimed for stability within and reproducibility across modalities. Stability was estimated by splitting the sample into halves 10,000 times. The similarity between the two halves was examined by computing the Adjusted Rand Index (ARI) between the two split partitions. To assess consistency, we generated 10,000 bootstrap samples for each modality and compared these samples between CBP modalities using the ARI (RSFC vs MACM, RSFC vs SC, MACM vs SC). An ARI value of 1 indicates that the clusterings are identical and a value of 0 suggests that the clusterings are not similar to each other, whereas negative values indicate a dissimilarity of clusterings higher than chance (see Supplemental material I.6 methods for detailed information).

\subsection{Evaluating denoising performance}

To better understand the actual effect of denoising strategies on the stability and consistency of RSFC-CBP partitions, we investigated the effect of denoising on seed voxels' time-course similarity and on connectivity profile dissimilarity. We assumed that structured noise influences the BOLD-response in the measured time-series in such a way that the voxels become more artificially similar (higher time-series similarity) and 
show higher similarity in their connectivity profiles. Accordingly, we can expect that a denoising method, which reduced structured noise successfully, will decrease timeseries similarity and increase dissimilarity of the connectivity fingerprint. Since this latter marker directly drives the clustering pattern, its sensitivity to denoising is crucially relevant in the CBP application perspective. We could indeed expect that efficient denoising would to some degree enhance voxels dissimilarity facilitating the assignment of voxels to clusters. We therefore first examined voxels similarity regarding their time-series as measured by correlations of the time-series (Pearson's correlation), but we also examined the dissimilarity of the seed voxels regarding their pattern of connectivity with all other brain grey matter voxels by computing the Euclidean distance between seed voxels' connectivity fingerprint.

\subsection{Consensus clustering}

In order to create a cross-modal and stable map of the hippocampus from functional modalities, we used the bootstrap resampling method (Bellec et al. 2010). The basic idea was to simulate the replication process of parcellation a large number of times to preserve stable features and to reduce the occurrence of unlikely or unstable individual patterns (Bellec et al. 2010). After having created 10,000 bootstrap samples containing a matrix with all seed voxels and their cluster' labels (assigning each voxel to a cluster for each modality), we pooled these samples. After pooling we identified for each seed voxel the most frequent assignment to a cluster by computing the mode. This procedure allowed each modality to be represented in the same regard independent of group or filter size, but only the most stable partition across both modalities was retained. Accordingly, if one modality provides unstable partitions, which is particularly likely for 
RSFC-CBP, the stable partitions from the other modality (here MACM) will determine the final clustering. Thus, this procedure promotes a final general parcellation, which is both, functionally cross-modal, and stable.

\subsection{Cluster characterization with BrainMap and NeuroSynth databases}

To characterize the clusters of our cross-modal consensus parcellation behaviorally we used two different databases, BrainMap (http://www.brainmap.org/) and NeuroSynth (http://neurosynth.org/). Both databases are complementary so that we expect their combination to provide novel insights into the behavioral association and the profile of a brain region (Genon et al. 2018a). Furthermore, using both databases circumvents a circularity limitation (see Supplementary methods I.2). In the BrainMap protocol, each activation peak has been individually labeled according to a predefined taxonomy of behavioral domains such as cognition.memory.working (see (Genon et al. 2017). Behavioral profiling was performed with a reverse inference approach (Genon et al. 2018a), which identifies the posterior probability $\mathrm{P}($ Task|Activation), that is the probability of task given activation in that cluster.

In contrast, studies in NeuroSynth were labeled according to terms occurrence in the paper by using a text-mining approach so that behavioral associations were determined by the terms used in the corresponding article text (Yarkoni et al. 2011). This automated strategy resulted in the inclusion of 11406 studies (tripled the number of archived studies in BrainMap), but can suffer from a lack of behavioral precision. We also used the reverse inference approach with NeuroSynth i.e. P(Term|Activation). The decoding with BrainMap was region of interest (ROI)-based whereas it was coordinate-based with NeuroSynth requiring the usage of centroid coordinates of each cluster in MNI152 space 
(see Supplementary Methods I.7, Table 1). The lexical meta-analysis approach of NeuroSynth required decision criteria for selecting functional associated terms so that we excluded all non-content words (e.g., "addressed", "abstract", or "reliable") and all brain terms that did not refer to function (e.g., "hippocampus", "middle temporal lobe"). Additionally, we pooled terms with the same phonological root (e.g. 'memory' was used as a generic term for 'memories'). All terms that reached a z-score higher than zero were included.

\section{Results}

To optimize first the reliability of RSFC-CBP, we examined the stability of its yielded partitions dependent on the application of different denoising strategies. After having identified the most reliable denoising method for RSFC, we investigated hippocampal organization across CBP modalities to determine consistency between modalities and levels of partition in order to establish a stable cross-modal functional map, which we characterized behaviorally in the last step.

\subsection{Stable RSFC parcellations as a function of denoising}

We measured stability within RSFC-CBP with split-half cross-validation and computed a two-way ANOVA with denoising as one factor (no denoising, GSR, WM/CSF, FIX, FIX+GSR vs FIX+WM/CSF), levels of partition as a second factor $(\mathrm{k}=2-7)$, and ARI as a dependent variable.

The analysis showed that the most robust RSFC parcellation was achieved when using $\mathrm{FIX}+\mathrm{WM} / \mathrm{CSF}(\mathrm{M}=0.82 \mathrm{ARI}, \mathrm{SE}=0.03), \mathrm{GSR}(\mathrm{M}=0.82 \mathrm{ARI}, \mathrm{SE}=0.03)$ or $\mathrm{WM} / \mathrm{CSF}$ 
regression $(\mathrm{M}=0.81 \mathrm{ARI}, \mathrm{SE}=0.04)$. The least stable parcellation was achieved when using FIX only $(M=.76$ ARI, $S E=.005)$; main effect denoising F $(5,719999)=60220, p<$ .0001] (see Figure 2a).

The main effect of partition levels was also significant $[F(5,719999)=7377.6, p<$ $.0001]$ demonstrating highest stability for 6 clusters $(\mathrm{M}=.81 \mathrm{ARI}, \mathrm{SE}=.03)$, followed by 7 clusters $(\mathrm{M}=.81 \mathrm{ARI}, \mathrm{SE}=.03)$. The ANOVA yielded also a significant interaction effect between denoising and levels of partition, $[F(25,719999)=6812.48, p<.0001]$ (see Figure 2d). All comparisons between denoising methods and between levels of partition were significant according to a post-hoc Bonferroni-corrected analysis $(p<0.001)$. However, the small magnitude of the differences between denoising approaches suggested that, overall, they all offered high stability. In the following section, we further investigated the effect of denoising at the voxel level to better understand how different methods influence voxels' properties and thereby the stability of the clustering.

Captions 


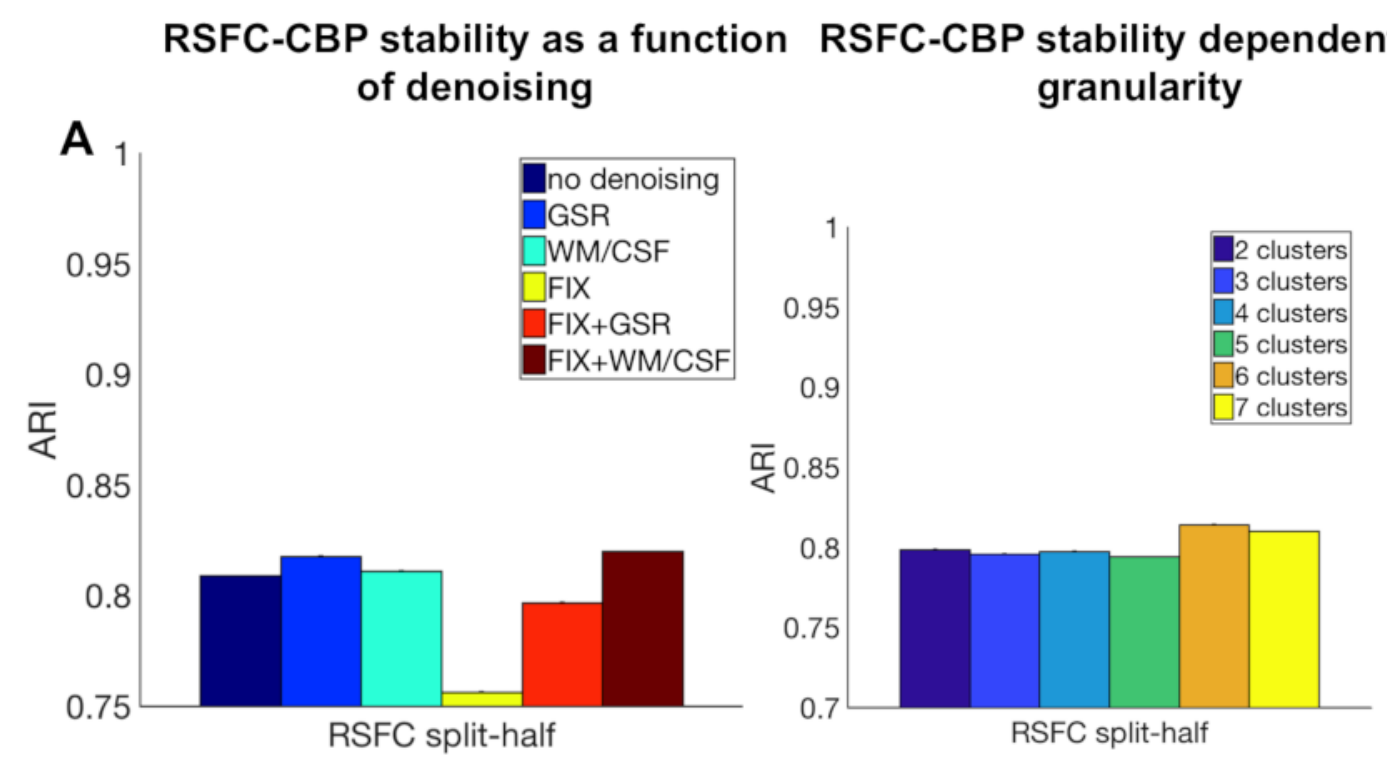

Effects of denoising on voxels' properties
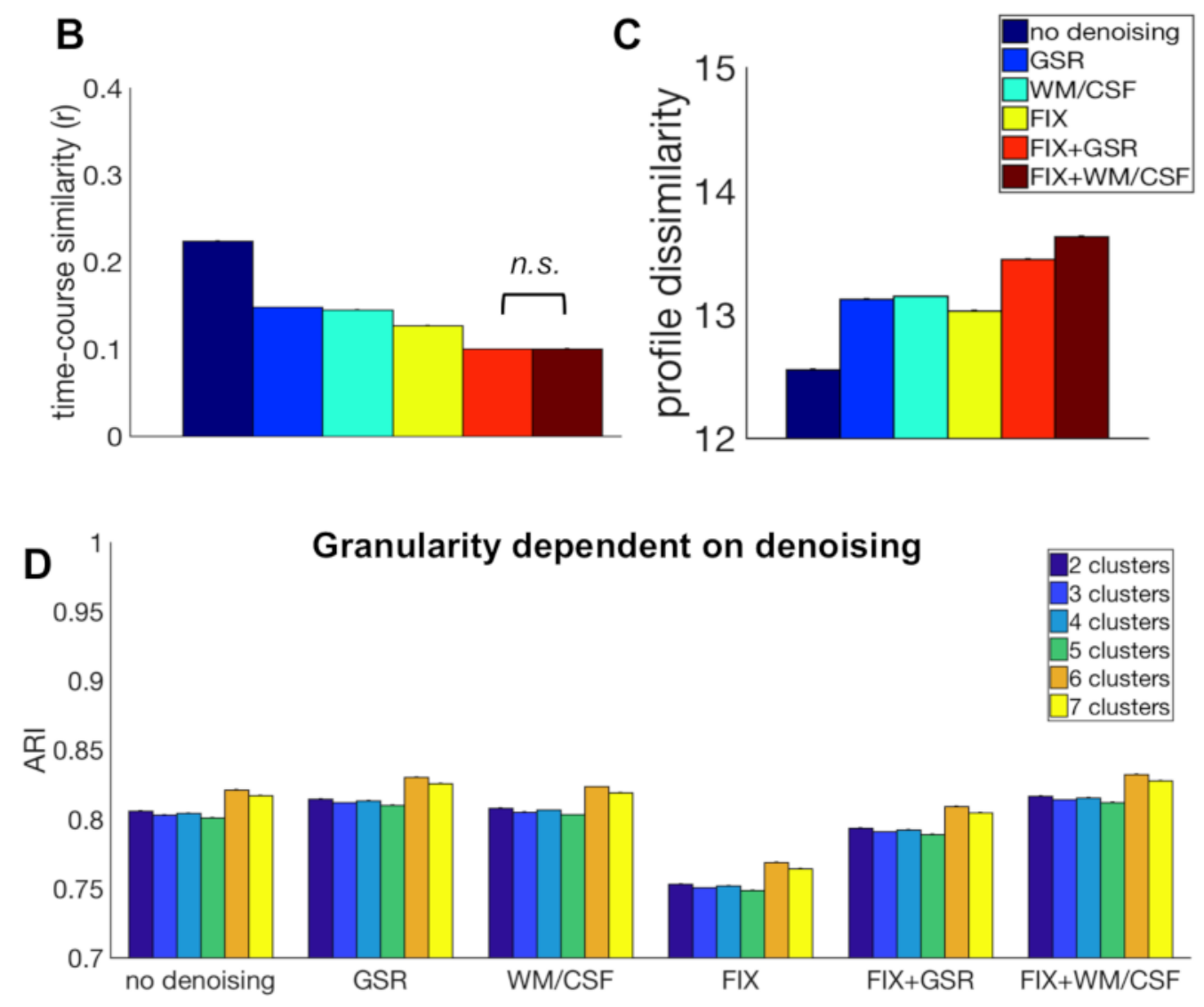

Figure 2. The effect of denoising on RSFC-CBP and on voxel functional properties.

A) Most stable hippocampal parcellations across all levels of partition $(\mathrm{k}=2-7)$ were 
obtained with FIX+WM/CSF, GSR and WM/CSF regression as denoising approaches. Bars indicate mean ARI ( \pm standard errors). Independent of denoising technique the highest stability was acquired for six clusters. All comparisons were statistically significant. B) Seed voxels' time-course similarity was reduced after the application of denoising. No significant difference was observed between FIX+GSR and FIX+WM/CSF whereas all the other comparisons were significant. C) Denoising resulted in an increase of seed voxels' dissimilarity in comparison to uncleaned data. FIX-related strategies demonstrated the strongest effect of connectivity profile dissimilarity. D) FIX+WM/CSF showed the highest stability across all levels of partition $(\mathrm{k}=2-7)$ compared to other denoising techniques. Mean ARI ( \pm standard errors). All comparisons were statistically significant.

3.2 Effects of denoising on voxels' time-course similarity and connectivity profile dissimilarity

We computed two separated ANOVAs in order to examine the effect of variance regression performed with denoising on each type of voxel measure separately: voxels' time-course similarity and voxels' connectivity profile dissimilarity.

The ANOVA with averaged seed voxels' time-course similarity as dependent variable revealed a significant main effect denoising $[\mathrm{F}(5,4311269)=78078.56, p<.0001]$, demonstrating a decrease in time-course similarity for denoised data. Post hoc Bonferroni-corrected multiple comparisons revealed no significant difference between FIX+GSR and FIX+WM/CSF regression $(p=.40)$, but all the other comparisons were significant $(p<.0001)$. The combination of a model-based (FIX) and model-free (GSR, 
WM/CSF) denoising strategy resulted in highly reduced seed voxels' time-course similarity compared to other techniques (Figure 2b).

The second ANOVA with averaged seed voxels' connectivity profile dissimilarity as dependent variable revealed an increase in dissimilarity of seed voxels with every additional denoising technique [significant main effect denoising: $F(5,4311269)=$ 113132.16, $p<.0001$ ] (Figure 2c). Our results showed that following denoising, seed voxels' connectivity profiles were more discriminable and especially, FIX+WM/CSF led to the highest dissimilarity between seed voxels' connectivity profiles. All post hoc Bonferroni-corrected comparisons were significant $(p<.0001)$.

Thus, overall our analyses supported the use of FIX+WM/CSF as a denoising strategy and accordingly this procedure was retained for the following multimodal functional parcellation.

\subsection{Robust levels of subdivisions across CBP modalities}

After defining the optimal denoising strategy (FIX+WM/CSF) from the voxels' properties, as well as from the parcellation's stability perspective, we examined which level of partition, in other words, granularity, promotes consistency across CBP modalities. We focused on the consistency measure instead of stability since we aimed to promote biological validity estimated through comparisons across modalities. To examine consistency across partition's levels, we computed one-way ANOVAs with number of clusters $(\mathrm{k}=2-7)$ as factor and ARI index measuring similarity as the dependent variable, separately for each pair of modality (RSFC vs MACM, RSFC vs SC and MACM vs SC). 
Cross-modal comparisons between SC and functional modalities (RSFC, MACM) resulted in less consistency than between the two functional CBP modalities (RSFC vs MACM). The highest similarity between RSFC and SC was achieved for a partition of $2(\mathrm{M}=.58$, $\mathrm{SE}=.03)$ and 6 clusters $(\mathrm{M}=.41, \mathrm{SE}=.05)[\mathrm{F}(5,119999)=167974.16, p<.0001](\mathrm{see}$ Figure 3). The comparison between MACM and SC revealed that the highest consistency occurred at a $2-(\mathrm{M}=.54, \mathrm{SE}=.15), 3-(\mathrm{M}=.47, \mathrm{SE}=.04)$ and 5 -cluster partition $(\mathrm{M}=.43$, $\mathrm{SE}=.06),[\mathrm{F}(5,119999)=17685.1, p<.0001]$. All post-hoc Bonferroni-corrected comparisons were significant $(p<.0001)$.

Visual examination suggested that the highest convergence between SC and functional modalities could be observed at low granularity, that is, for 2-cluster partition in which all modalities subdivided the hippocampus into an anterior and a posterior cluster. At the next subdivision level, partitions already differed markedly between modalities. Namely, the pattern of subdivisions based on SC is dominated by a medial-lateral organization that strikingly mimics cytoarchitecture differentiation between subiculum and CA subfields. Such a medial-lateral subdivision first concerns the posterior portion of the hippocampus (at 3-cluster partition) but extends into the hippocampus head at 4cluster partition (see Figure 3).

In turn, functional convergence between RSFC and MACM showed a significant effect on the $\operatorname{ARI}[F(5,719999)=12506.39, p<.0001]$, with the highest convergence being observed for partitions of $5(\mathrm{M}=.55, \mathrm{SE}=.10), 3(\mathrm{M}=.49, \mathrm{SE}=.03)$, and 7 clusters $(\mathrm{M}=$ $.48, \mathrm{SE}=.02$ )(see Supplementary results II.2, Table 2). Consequently, partitions into 3, 5 and 7 subregions were considered as optimal level of partitions for defining robust functional maps of the hippocampus.

In addition to the quantitative analysis, the visual examination of the partition schemes also proposed high convergence for 3, 5 and 7 clusters, revealing that the 3 -cluster 
partition divided the hippocampus into anterior, intermediate and posterior subregions in both modalities (Figure 3). In turn, the 5-cluster partition divided the hippocampus into one anterior cluster (head), three intermediate clusters (intermediate caudal, intermediate lateral and medial, for the body) and lastly, a posterior cluster (tail). Finally, the 7-cluster partition included three anterior, three intermediate clusters and one posterior subregion (see Figure 3). 

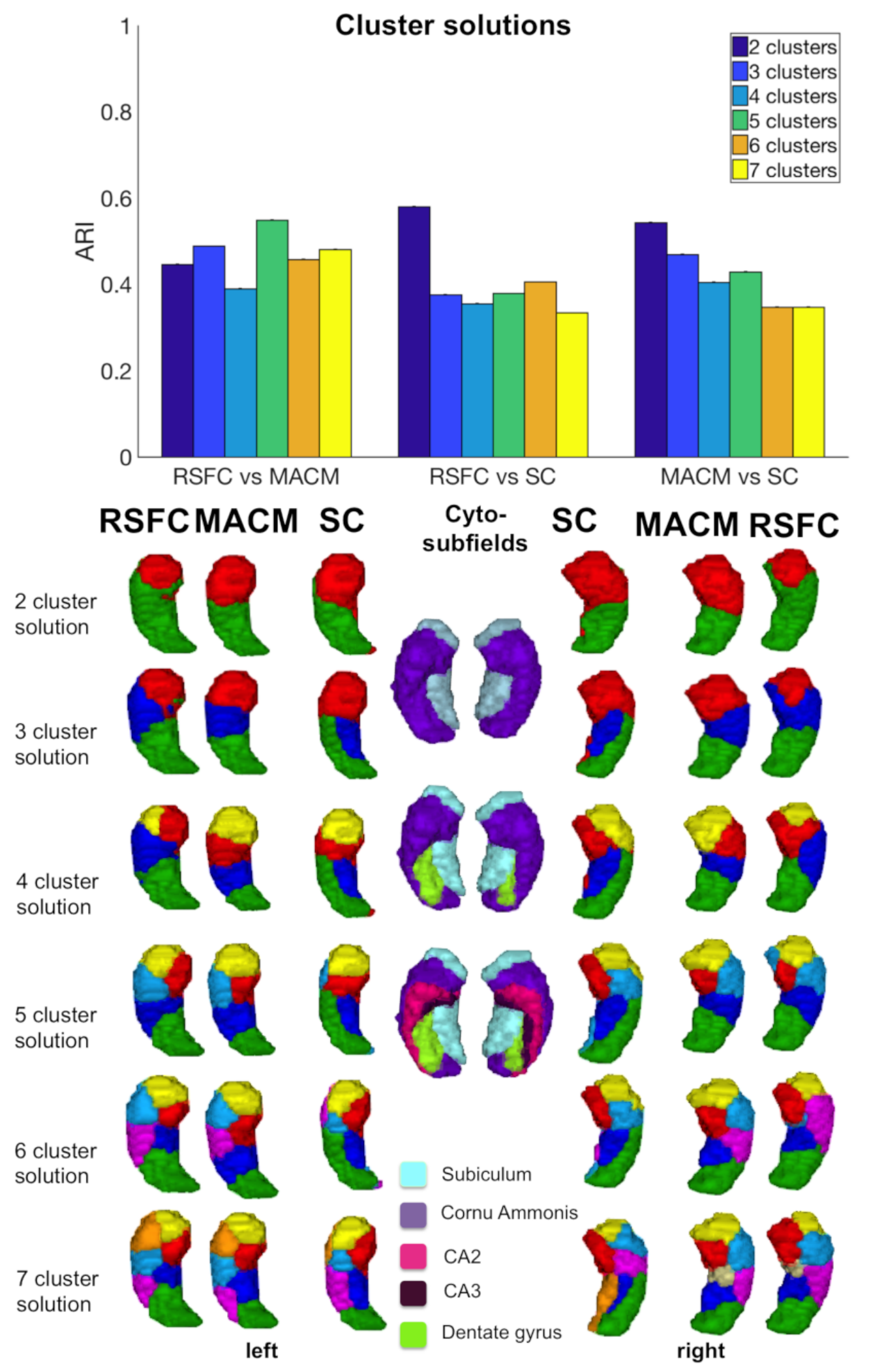

Figure 3. Hippocampus partitions based on SC, MACM, RSFC and cytoarchitecture mapping. Mean ARI ( \pm standard error). All comparisons between cluster solutions showed significant differences. 


\subsection{Cross-modal functional consensus map}

Cross-modal comparisons of CBP modalities revealed high convergence between RSFC and MACM, especially at higher granularities, whereas SC showed an idiosyncratic subdivision that deviated from pure functional modalities. For this reason we established a pure functional cross-modal map of the hippocampus using bootstrap resampling as described in section 2.9 and based on RSFC and MACM parcellations while excluding SC. Importantly, we created functional maps at different granularity levels (3,5 and 7 clusters) reflecting convergence between modalities as an approximation of biological validity. These functional maps at different partition levels should allow the community to investigate hippocampus' function and dysfunction at various levels of organization. In the present study, we focused on the 7 cluster partition to study hippocampus function as this high level of partition offers a detailed architecture along the anterior-posterior axis with small functional units.

As illustrated in Figure 4, each and every retained level of granularity revealed a specific aspect of hippocampal functional organization. The cross-modal 3-cluster partition subdivided the left and right hippocampi into an anterior (head), intermediate (body) and a posterior (tail) subregion. At the next subdivision (5-cluster granularity), bilateral hippocampi were partitioned into a posterior, intermediate part including 3 subregions - intermediate caudal, intermediate lateral rostral and intermediate medial rostral -, and finally an anterior subregion. The 7-cluster cross-modal partition showed hemispheric asymmetry. The body of the right hippocampus was subdivided into one intermediate lateral and two intermediate medial clusters. In contrast, the left hippocampus was partitioned into two intermediate lateral clusters and one 
intermediate medial cluster (see Figure 4). However, the posterior tail-cluster as well as the head, which was subdivided into three clusters (anterior rostral, anterior lateral and anterior medial), were found in both hemispheres.

We hypothesized that the subdivision into medial vs lateral subregions could partially reflect the already known cytoarchitectonic subdivision. The lateral segments in the body of the hippocampus corresponded mainly to the CA1-3 subfields and the medial clusters mainly to the subiculum. This hypothesis was supported by a quantitative comparison of our clusters with cytoarchitecture from the Anatomy Toolbox (see Table 1). 


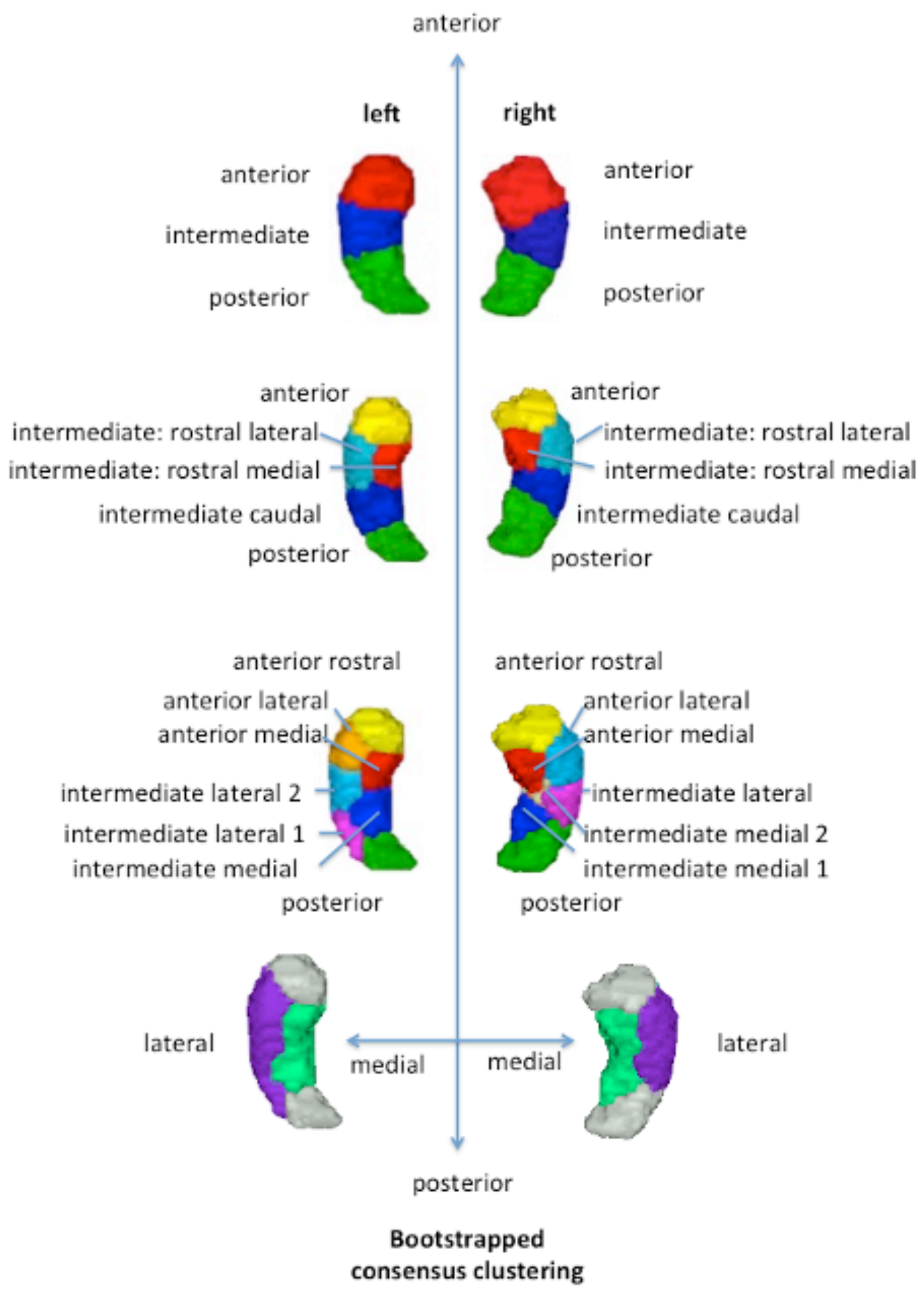

Figure 4. Functional multimodal maps across different granularities showing differentiation along the anterior-posterior and the medial-lateral dimension. 
Table 1 Consensus hippocampus in comparison to cytoarchitecture

\begin{tabular}{|c|c|c|c|c|c|}
\hline Consensus cluster & $\begin{array}{l}\text { Cluster } \\
\text { size }\end{array}$ & $\mathrm{x}$ & $\mathrm{y}$ & $\mathrm{z}$ & $\begin{array}{l}\text { Overlap with } \\
\text { cytoarchitectonic } \\
\text { subfields }\end{array}$ \\
\hline \multicolumn{6}{|l|}{ Right hippocampus } \\
\hline $\begin{array}{l}\text { Anterior rostral cluster } \\
\text { (yellow) }\end{array}$ & 161 & 22 & -10 & -24 & CA1, Subiculum \\
\hline $\begin{array}{l}\text { Anterior lateral cluster } \\
\text { (light blue) }\end{array}$ & 181 & 31 & -16 & -20 & $\begin{array}{l}\text { CA1, DG, Subiculum, } \\
\text { CA2 }\end{array}$ \\
\hline $\begin{array}{l}\text { Anterior medial cluster } \\
\text { (red) }\end{array}$ & 139 & 21 & -17 & -17 & Subiculum, CA3 \\
\hline $\begin{array}{l}\text { Intermediate lateral cluster } \\
\text { (purple) }\end{array}$ & 128 & 33 & -26 & -12 & $\mathrm{DG}, \mathrm{CA} 1, \mathrm{CA} 2$ \\
\hline $\begin{array}{l}\text { Intermediate medial } 2 \\
\text { cluster (ocher) }\end{array}$ & 34 & 26 & -22 & -16 & Subiculum \\
\hline $\begin{array}{l}\text { Intermediate medial } 1 \\
\text { cluster (dark blue) }\end{array}$ & 86 & 24 & -31 & -9 & Subiculum, DG \\
\hline Posterior cluster (green) & 129 & 26 & -37 & -2 & $\mathrm{DG}, \mathrm{CA} 1$ \\
\hline \multicolumn{6}{|l|}{ Left hippocampus cluster } \\
\hline $\begin{array}{l}\text { Anterior rostral cluster } \\
\text { (yellow) }\end{array}$ & 166 & -23 & -11 & -24 & CA1, Subiculum \\
\hline $\begin{array}{l}\text { Anterior lateral cluster } \\
\text { (orange) }\end{array}$ & 122 & -31 & -15 & -21 & CA1, DG, CA2 \\
\hline $\begin{array}{l}\text { Anterior medial cluster } \\
\text { (red) }\end{array}$ & 149 & -24 & -20 & -18 & Subiculum, CA3 \\
\hline $\begin{array}{l}\text { Intermediate lateral } 2 \\
\text { cluster (light blue) }\end{array}$ & 133 & -33 & -24 & -14 & DG, CA1, CA2 \\
\hline $\begin{array}{l}\text { Intermediate lateral } 1 \\
\text { cluster (purple) }\end{array}$ & 76 & -31 & -35 & -7 & DG, CA1 \\
\hline
\end{tabular}




\begin{tabular}{llllll}
\hline $\begin{array}{l}\text { Intermediate medial cluster } \\
\text { (dark blue) }\end{array}$ & 112 & -26 & -30 & -9 & Subiculum, DG \\
$\begin{array}{l}\text { Posterior cluster (green) } \\
\text { Pa }\end{array}$ & -20 & -35 & 1 & DG, CA1 \\
\hline
\end{tabular}

\subsection{Cluster characterization}

After having defined a functional parcellation map of the hippocampus we characterized the subregions with regard to behavioral functions using BrainMap and NeuroSynth activation databases. We focused on the finer partitions ( 7 subregions) since an examination of changes in behavioral associations across subregions at this high partition level could provide novel insights into the functional dimensions, which has not been investigated previously. More concretely, at this high level of partitions, we could both track behavioral associations across the anterior-posterior gradient and explore medial-lateral differentiation.

\subsubsection{Anterior vs. posterior functional differentiation}

The characterization with BrainMap and NeuroSynth revealed a functional gradient along the anterior-posterior axis of the hippocampus that, on the one hand, supported the hypothesis of an emotion-cognition gradient, and on the other hand, suggested a self-world centric processing gradient.

In BrainMap anterior clusters were more likely engaged in emotion processing whereas posterior subregions displayed a sparse functionality emphasizing higher cognition functions requiring abstract representations (e.g. Cognition.memory, Cognition.memory.explicit, Cognition.memory.language.semantics). (see Figure 5). In 
addition, the anterior clusters were associated with various behavioral domains such as perception, interoception and cognition, demonstrating a diverse behavioral spectrum. NeuroSynth provided a more detailed functional distinction along the anterior-posterior axis suggesting a gradient from self-centric (anterior parts) to more world-centric processing (posterior parts) as represented in Figure 6. The more anterior head clusters were engaged in cognitive and emotional processes related to personal experiences (e.g. episodic memory, experiences, autobiographical memory), whereas the more posterior clusters were associated with behavior like navigation (which requires the use of an abstract representation) and the processing of information in its environmental context. All the other intermediate-body and head subregions showed a graduated profile within this qualitative behavioral gradient (see Figure 6) and all clusters, independent of their position along the anterior-posterior axis, were associated with encoding, memory and retrieval processes. Interestingly, the gradients were especially evident in the lateral clusters (green, purple, light blue (orange) and yellow) of the hippocampus that were associated with CA subfields along the anterior-posterior axis as illustrated in Figure 5 and 6. 
BrainMap Domains: left hippocampus

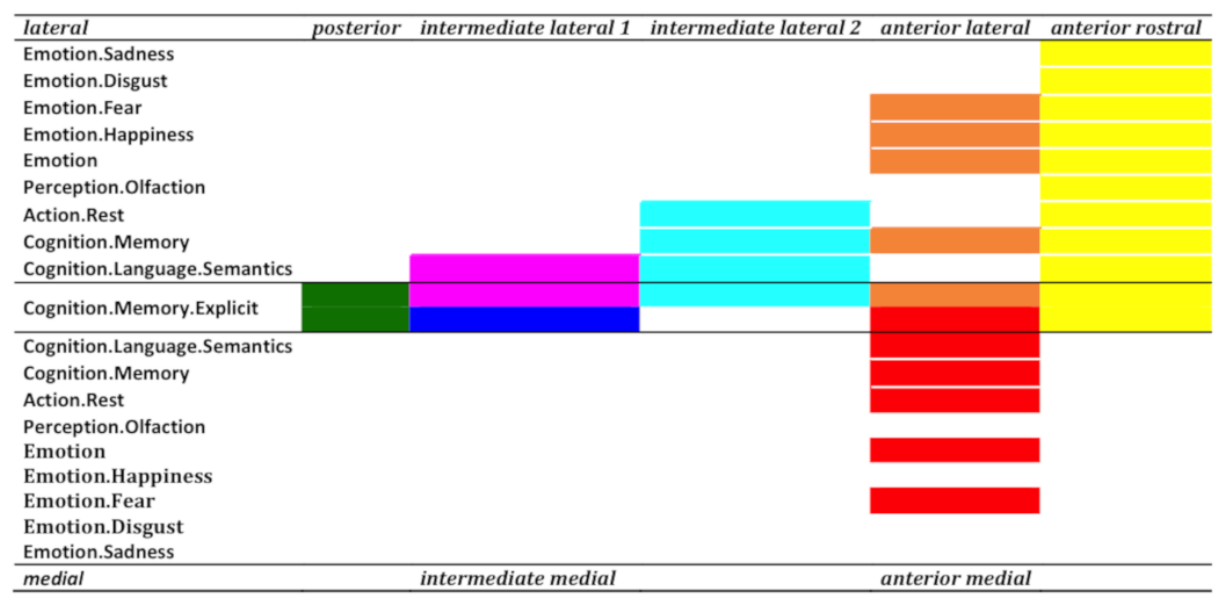

Emotion/ anterior

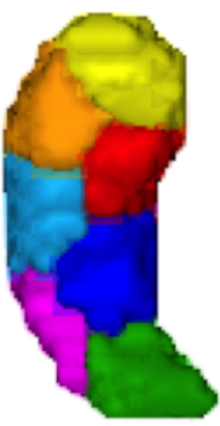

Cognition/ posterior

BrainMap Domains: right hippocampus

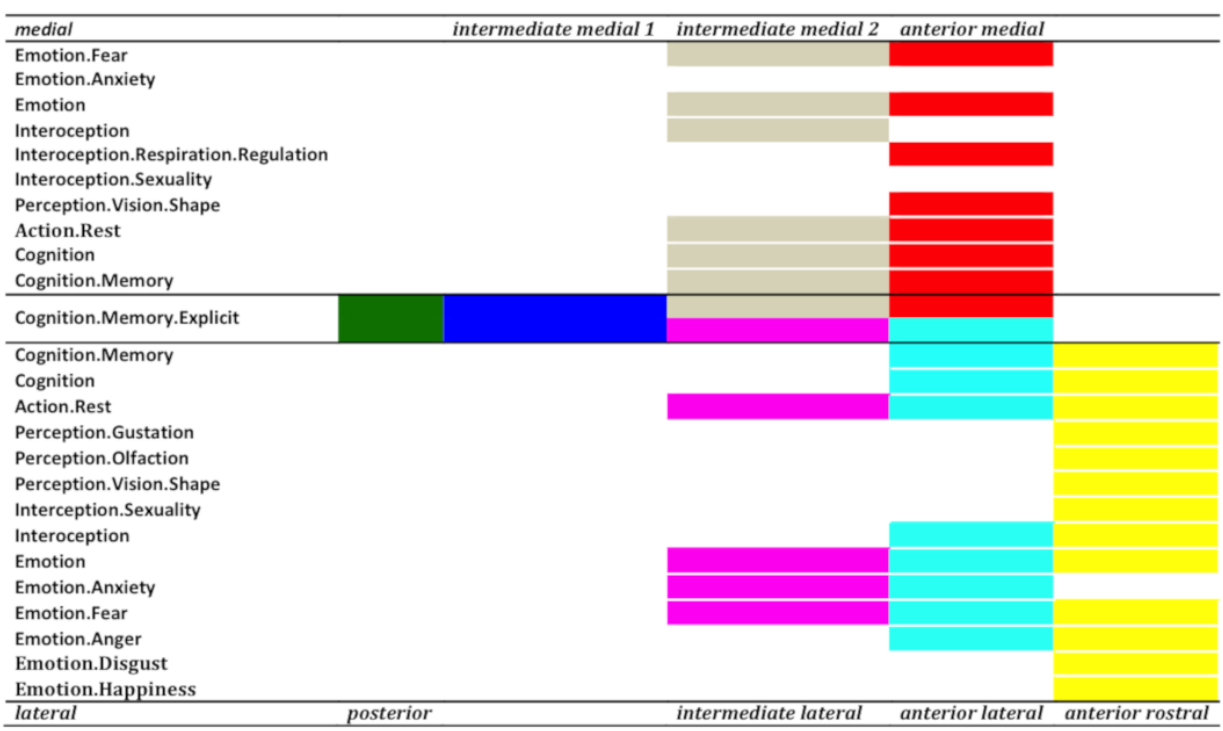

Cognition/ posterior

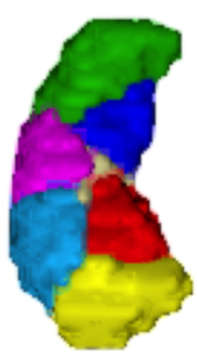

Emotion/ anterior

\section{Figure 5. Anterior to posterior characterization with BrainMap.}


Neurosynth characterization: left hippocampus
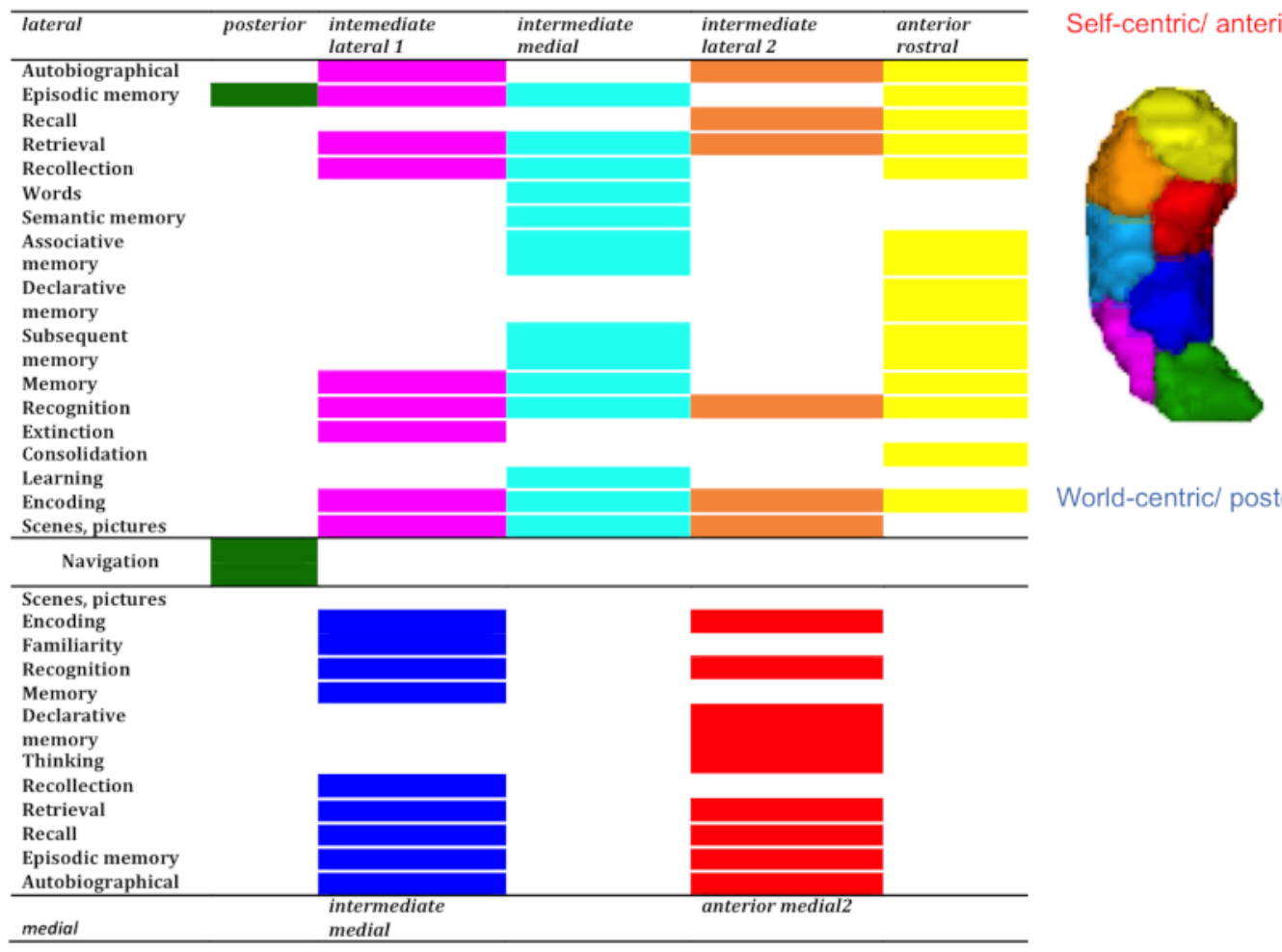

World-centric/ posterior

Neurosynth characterization: right hippocampus

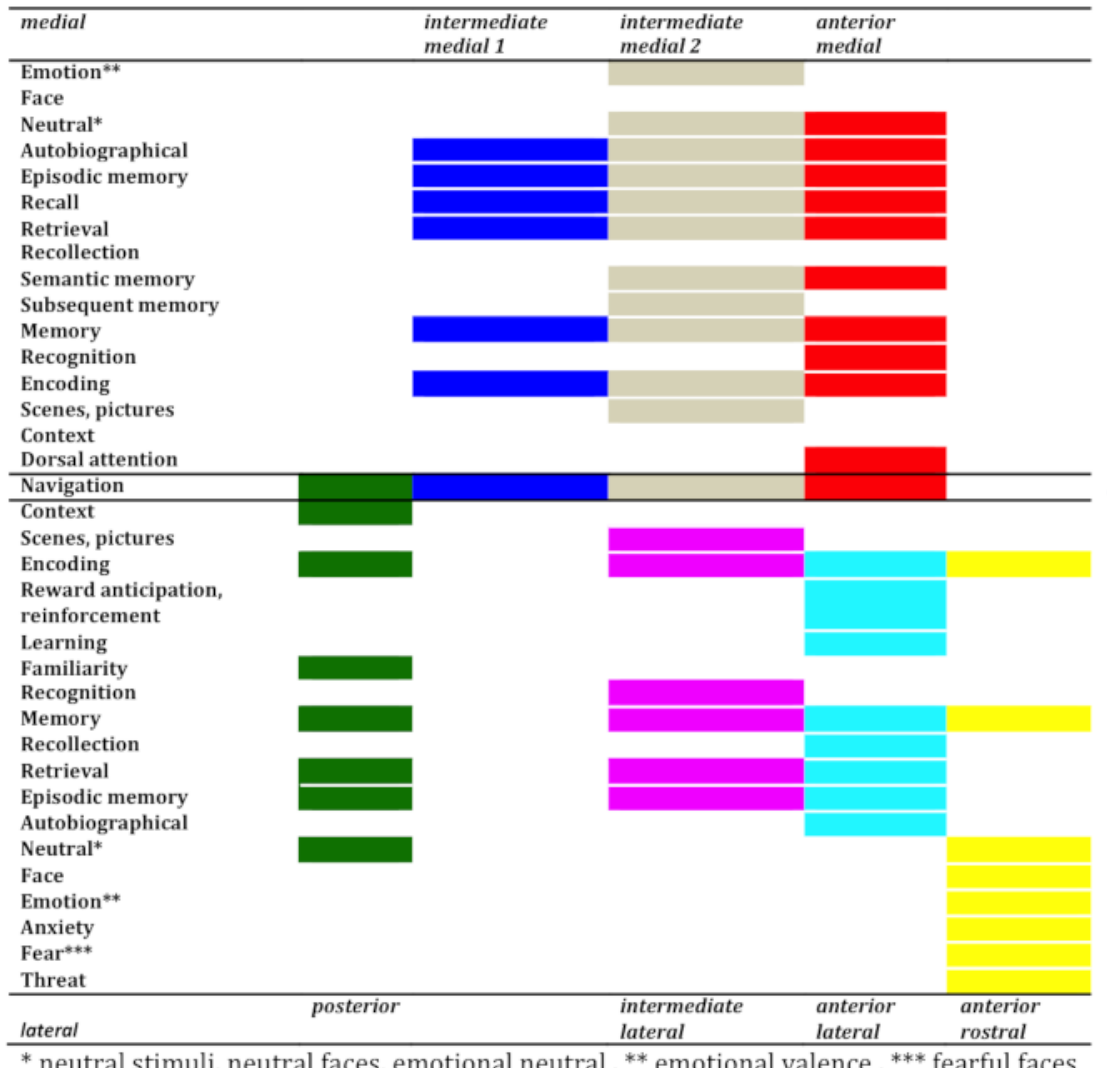

amiliarity

Recognition

Episodic memory

utobiographica

motion*

Anxiety

Threat

* neutral stimuli, neutral faces, emotional neutral, ${ }^{* *}$ emotional valence, ${ }^{* * *}$ fearful faces

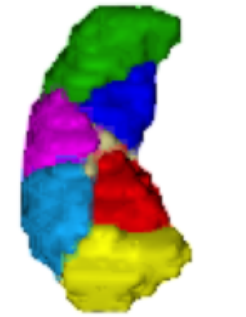

Self-centric/ anterior

Figure 6. Anterior to posterior characterization with NeuroSynth. 


\subsubsection{Medial vs. lateral functional differentiation}

In addition to an anterior-posterior organization the parcellation yielded a medial vs lateral differentiation predominantly in the body and partly in the head. In order to explore the functional relevance of the medial-lateral axis, we merged the intermediate clusters into one medial VOI segment and one lateral VOI segment, while anterior rostral and posterior subregions were not integrated (see Figure 4). Along the medial-lateral dimension the functional differentiation was less obvious with only slight differences between the two segments (see Figure 5 and 6). The medial segments were engaged in perception (visual shape discrimination), interoception (respiration regulation), dorsal attention and familiarity. Navigation, declarative memory and thinking were also associated with medial parts. In contrast, the lateral segments seemed to assimilate information into the hippocampal memory-system hence being engaged in associative memory, learning, reinforcement and extinction. Finally, we observed a lateralization effect in the sense that left lateral parts were involved in words and language processing and the right lateral subregions in emotion processing of happiness, anger and anxiety.

\section{Discussion}

In this study, we investigated the hippocampal organization in humans bridging the gap between hippocampal architecture and function using multi-modal CBP and two complementary databases for functional characterization. In contrast to other CBP modalities, RSFC, which is especially sensitive to noise, required a preliminary optimal denoising, which we evaluated in this study in regard to clustering stability and voxels' 
properties. Our results showed that the combination of a model-based (FIX) and a model-free (WM/CSF regression) denoising technique resulted in stable and biological plausible parcellations estimated through convergence across modalities. Especially, both pure functional modalities, MACM and RSFC, displayed high convergence at lower and higher parcellation granularities and could therefore be combined to derive a crossmodal functional map. We excluded SC from the cross-modal map as this modality demonstrated a relatively specific organization partly reflecting functional, as well as micro-architectonic characteristics. We emphasized and characterized the cross-modal seven-cluster-hippocampus yielding a subdivision into one posterior cluster, three head clusters and depending on lateralization two or three intermediate clusters along the anterior-posterior and medial-lateral dimension. Following this, our behavioral profiling of the clusters revealed a functional emotion-cognition and a self-world centric gradient along the anterior-posterior dimension, which seemed to be particularly evident along the lateral subregion and more pronounced in the right hemisphere. In the following sections, after briefly discussing our new methodological findings regarding denoising for RSFC-CBP, we discuss the new insight into hippocampus organization and function brought by the current study with regard to previous literature.

\subsection{Optimal denoising for RSFC-CBP}

Our preliminary goal was to find a stable and consistent RSFC parcellation. But in the absence of unanimous guidelines of denoising approaches for RSFC-CBP in the scientific literature, we investigated various strategies with regard to stability, and voxels' properties such as time-course similarity and connectivity profile dissimilarity. 
FIX+WM/CSF was found to contribute to highly stable partitions of RSFC-CBP, although other denoising techniques showed likewise high stability of parcellations. The subsequent examination of voxels' properties on which the clustering builds suggested two potential mechanisms underlying higher stability of FIX+WM/CSF. First, the part of variance neutralized by FIX+WM/CSF seemed to contain structured noise, as seed voxels' time-course similarity highly decreased when this strategy was applied (when compared to not denoised data and other denoising strategies, except for FIX+GSR). Secondly, and more importantly in the application-driven perspective, FIX+WM/CSF increased the discrimination between voxels as reflected by the significant improvement of seed voxels' connectivity profile dissimilarity. These influences eventually resulted in a better assignment of voxels to clusters. Overall our investigation promoted the combination of a model-based (FIX) and a model-free (WM/CSF regression) technique as an optimal denoising method, both from voxel-wise properties and partition-wise clustering. Burgess et al. (2016) already proposed to use FIX and GSR simultaneously in order to eliminate both local spatial artifacts and global drifts in fMRI data. Our results also suggested that the combination of FIX and GSR successfully removed structured noise outperforming FIX or GSR applied separately. FIX+GSR also led to stable parcellations in a similar extent than FIX+WM/CSF, but the use of FIX+WM/CSF was further supported by its improvement of voxels connectivity fingerprint discriminability, which is especially important in the clustering context. The reason why FIX+GSR performed less efficiently might be that GSR on one hand effectively neutralized motion artifacts, but on other hand, distorted distance relationships (Murphy et al. 2009; Power et al. 2014; Satterthwaite et al. 2017; Satterthwaite et al. 2013; Yan et al. 2013) that influenced connectivity measures. Based on these considerations, we can assume that GSR can have detrimental effects for parcellation 
and that WM/CSF represents a better alternative for combination with FIX. It has been suggested that WM/CSF regression eliminates more effectively respiration and cardiac effects (Anderson et al. 2011; Jo et al. 2010; Liu 2016) and more generally, any slow undulations compared to GSR or FIX. FIX, in turn, could catch local or spatial related artifacts, which cannot be captured by WM/CSF in the same way. For these reasons, we here suggest that the combination of FIX with WM/CSF represents the most sophisticated double-approach for denoising, in particular in the context of CBP.

4.2 A convergent functional topography of the hippocampus across different measures of functional connectivity

In our study, two functional CBP modalities, task-independent (RSFC) and taskdependent (MACM), yielded CBP results with high convergence at the granularity of 3-, 5- and 7-cluster partitions, despite divergent methodological procedures. This high similarity between conceptually related methods, but based on completely different procedures and independent objects of investigation (i.e. co-activations across paradigms vs participants' RS-fMRI) argued for biological relevance of the revealed topographical pattern. Importantly, the convergence in partition scheme between the two modalities can not be attributed to an artifact intrinsic to the k-means clustering as a similar clustering procedure applied to structural covariance data revealed a different partition scheme. Indeed, SC-CBP parcellations deviated substantially from functional organizations already at low granularity even though at high granularity this modality also contained a functional head separation, the medial-lateral differentiation within body and tail seemed to mirror cytoarchitectonic differentiation between cornu ammonis and subiculum. Although our goal was not to elucidate the relationship 
between functional aspects, microstructure and SC, our parcellation work suggested that SC pattern could to a greater extent than functional connectivity be influenced by microstructural aspects. Future studies should further investigate the relationships between SC, microstructure and functional connectivity across the human brain.

Overall, our findings converged with previous literature reporting studies in different methods and species, which further supported the biological validity of the obtained parcellations. In this context, one of the most prominent subdivisions for the hippocampus along the anterior-posterior axis is the tripartite model found in human and non-human segmentations. According to that model, the hippocampus is subdivided in an anterior (ventral, head), intermediate (body) and posterior (dorsal, tail) subregion, shown by anatomical (Swanson and Cowan 1977) and gene expression data in rodents (Dong et al. 2009), and CBP research in humans (Robinson et al. 2015; Robinson et al. 2016).

Furthermore, Robinson et al. (2015) and Chase et al. (2015) extended the subdivision of the hippocampus and revealed an organization into five subregions for the entire hippocampus as well as for subiculum subfield using CBP. Robinson's (2015) MACM parcellation yielded four serial clusters along the anterior-posterior axis and a fifth anterior head-cluster tilted medially (see Figure 1). Of note, this pattern was replicated in the current study by RSFC parcellation but does not appear as a prominent pattern retained in the cross-modal map. Beyond these minor differences between studies, nonspecies studies divided the CA1 subfield in rodents in five serial segments along the dorsoventral axis (Petrovich et al. 2001; Risold and Swanson 1996), supporting the observation and potential biological meaningfulness of serially aligned clusters in our and Robinson's work. 
To investigate hippocampus' function, we capitalized on the consensual 7-cluster partition scheme as it provides a very detailed functional architecture representing anterior-posterior gradient into small units, which was never achieved before. Our cross-modal map of the hippocampus at this level exhibited three head clusters, three or two intermediate clusters dependent on lateralization and one tail cluster. Interestingly, the posterior cluster in the tail remained as a relatively homogeneous functional region across functional modalities and granularities. This level of fine parcellation also contains a medial-lateral differentiation, which seemed to reflect differences between cornu ammonis and subiculum, respectively. According to the current parcellation, these two regions could be partitioned into serially positioned clusters along the anteriorposterior axis, which is in line with other studies (Dong et al. 2009; Fanselow and Dong 2010). In other words, our clustering of seven subregions seemed to reflect on one hand the differentiation between cornu ammonis and subiculum and on the other hand further subdivisions along the anterior-posterior axis.

\subsection{Functional organization of the hippocampus and human behavior}

Based on the high convergence between RSFC and MACM we computed a fine consensual parcellation combining both modalities. We then drew up the behavioral profile of each subregion using BrainMap and NeuroSynth as two complementary databases. We hence behaviorally characterized the anterior-posterior gradient and the medial-lateral differentiation taking an overarching view with activation databases.

\subsubsection{Medial-lateral differentiation}


We hypothesized that our organization along the medial-lateral axis reflected the differentiation between the subiculum (medial) and the CA subfields (lateral) evidenced by cytoarchitecture. Our behavioral profiling suggested that the medial segments participated in navigation, declarative memory and familiarity, whereas the lateral segments were associated with reinforcement, learning and extinction. Overall functional differences along the medial-lateral axis were sparse. Based on these behavioral descriptions we can only speculate that the lateral clusters were functionally involved in storing potentially integrating information into other systems and networks, whereas the functional specificity of the medial subregion was less evident. The conceptual inferences of the present study are limited on one hand by the spatial precision of standard MRI measurements and on the other hand, by current cognitive ontologies which have been derived by the study of human behavior and mind. By making all our partitions openly available to the scientific community, we invite future studies to further complement these first integrative findings on hippocampus organization and function. Nevertheless and importantly, the medial-lateral differentiation in the current fine parcellation has revealed that the functional gradient proposed in previous studies is mainly evident along the lateral segment. This aspect of functional organization of the hippocampus has presumably complicated or obscured the characterization and understanding of the gradient. In the current study, extensive behavioral profiling of fine subdivisions has allowed us to discuss new hypothesis beyond common psychology distinctions of behavioral functions.

\subsubsection{Anterior-posterior organization}


Evidence for an emotion-cognition and self-world centric gradient

The present study brought new integrative insights across research fields on the longitudinal functional differentiation of the hippocampus previously demonstrated in rats (de Hoz et al. 2003; Jung et al. 1994; Moser et al. 1993; Moser and Moser 1998; Vann et al. 2000), monkeys (Colombo et al. 1998) and humans (Poppenk et al. 2013; Robinson et al. 2015; Robinson et al. 2016; Small et al. 2001) by revealing an emotion-cognition gradient within the broad behavioral domains of BrainMap and a self-world centric gradient based on more specific behavioral concepts in NeuroSynth. For reader's convenience the main pattern is illustrated in Figure $\mathbf{7}$ in which we focused on lateral clusters. Below we discuss these patterns with regard to previous hypotheses proposed in the literature.

Besides the already often discussed differentiation of emotion-cognition along the anterior-posterior dimension, that we replicated with both databases integrating the scientific knowledge of thousand of studies, we also speculated that hippocampal organization along the anterior-posterior axis could be better explained with a selfworld centric gradient. As actually almost all subregions are associated with memory processes, we speculate a self-centric information processing mode in the most anterior cluster with psychological functions such as autobiographical memory and emotion (see Figure 7) contrasting with a world-centric processing of information calling concepts such as navigation, scene and context processing associated with the most posterior hippocampal subregions. In other words, the overall pattern of behavioral concepts along the anterior-posterior axis suggest, in our view, a latent or underlying functional change from one pole of self-related processing to another pole of world-related processing. Importantly, general processes like encoding, and retrieval appeared equally 
distributed along the longitudinal axis. Thus, altogether our findings are more in favor of a self-world centric information processing gradient rather than a behavioral domainwise (imagination-perception (Zeidman and Maguire 2016) or encoding-retrieval organization (Kim 2015; Lepage et al. 1998; Prince et al. 2005)) along the anteriorposterior axis of the hippocampus. Importantly, this self- and world-centric distinction may be reminiscent of egocentric vs allocentric distinction suggested by studies of spatial processing in rodents (Morris et al. 1986) and is particularly evident in the right hemisphere, but their meaning in the human cognitive system should nevertheless be considered beyond spatial representation, that is, also in relation to memory and decision making domains. If this hypothesis holds true, it has important implications for our understanding of psychiatric and neurological disease but its validity remains to be further evaluated in future studies using hypothesis-based experiments. 


\begin{tabular}{|c|c|c|c|}
\hline \multirow{2}{*}{\multicolumn{4}{|c|}{ postertor Intermeaiate Iateral intermealate Iateraiz antertor Taterat anterior rostrat }} \\
\hline & & & \\
\hline \multicolumn{4}{|l|}{ Emotion.Disgust } \\
\hline \multicolumn{4}{|l|}{ Emotion.Fear } \\
\hline \multicolumn{4}{|l|}{ Emotion. Happiness } \\
\hline \multicolumn{4}{|l|}{ Emotion } \\
\hline \multicolumn{4}{|l|}{ Perception.Olfaction } \\
\hline \multicolumn{4}{|l|}{ Action.Rest } \\
\hline \multicolumn{4}{|l|}{ Cognition.Memory } \\
\hline Cognition.Language.Semantics & & & \\
\hline Cognition.Memory.Explicit & & & \\
\hline
\end{tabular}

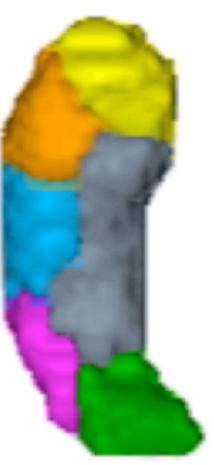

Neurosynth characterization: right hippocampus
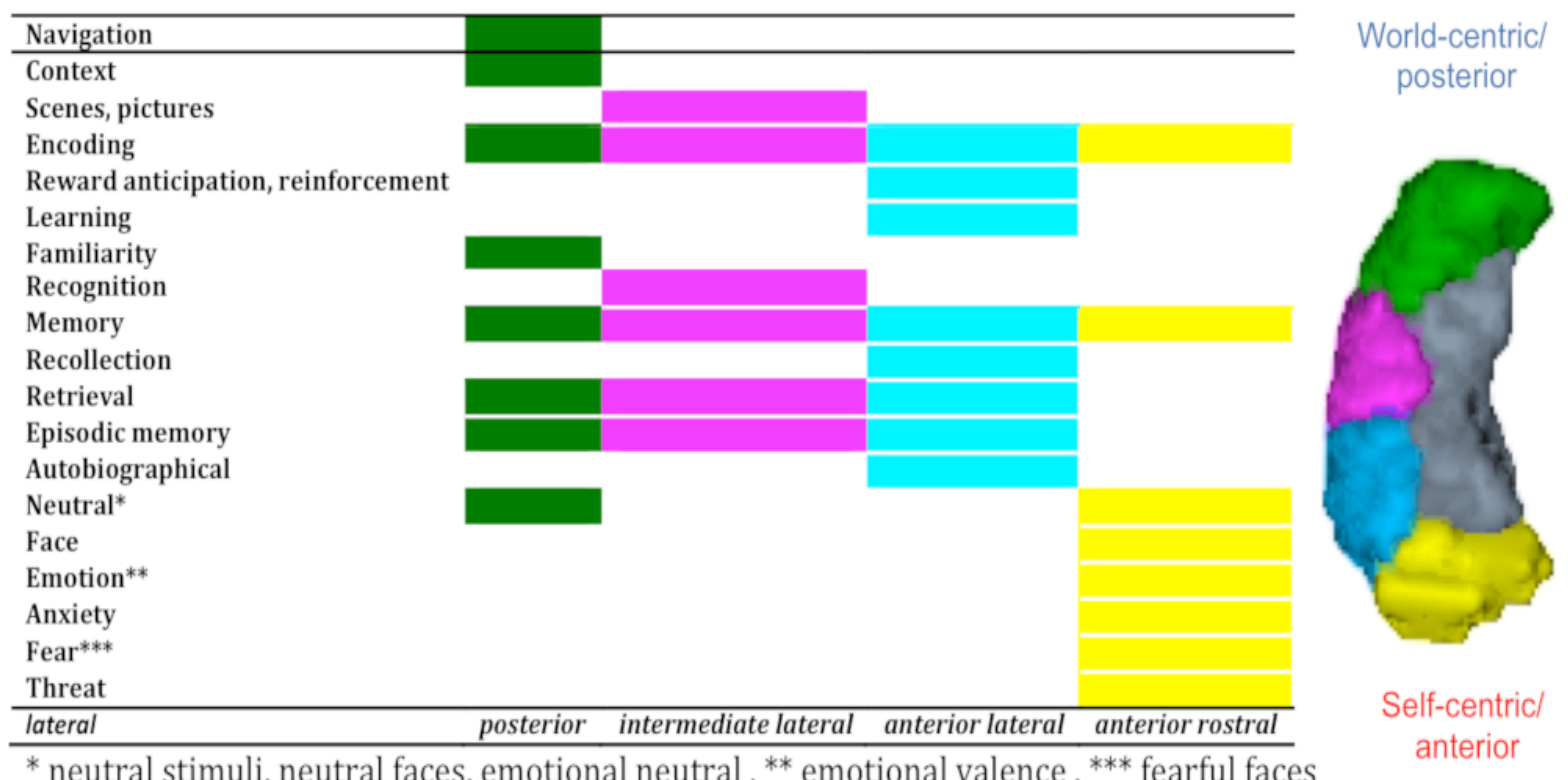

Self-centric/ anterior

Figure 7. Emotion-cognition and self-world centric functional gradient along the anterior-posterior axis. Lateral clusters display an emotion-cognition gradient yielded with BrainMap and a self-world centric gradient found with NeuroSynth.

\section{Limitations}

The large-scale data aggregation on which the current study capitalized also comes with specific limitations. First, we focused on MRI, a method, which has a relatively limited 
spatial resolution and a relatively limited signal-to-noise ratio in the subcortical structures. Therefore, the clusters we have obtained can only be considered as homogeneous regions with respect to the usual MRI signal. Accordingly, we assume that our lateral segment actually represents an aggregation of the known different CA subfields showing different cytoarchitecture and function. In particular, rodent studies suggested that CA1 and CA3 differ in their involvement in processes such as pattern separation and pattern completion (Guzowski et al. 2004). While these differentiations remain debated in humans (Deuker et al. 2014; Koster et al. 2018) in whom processes such as encoding, retrieval and association between unrelated items have been additionally proposed to differentiate distinct subfields (Bakker et al. 2008; Deuker et al. 2014; Dimsdale-Zucker et al. 2018). These differentiations could not be investigated in the present study due to a lack of behavioral precision in the representative concepts of both activation databases, in addition to the aforementioned limited spatial resolution. Future studies should therefore investigate how the anterior-posterior functional differentiation could be integrated with the subfields functional specialization in the hippocampus.

Another relevant limiting point refers to the complex structure of the hippocampus itself and its consequences for the optimal number of clusters. The human hippocampus is characterized by angulation and a variable number of digitations (Ding and Van Hoesen 2015; Treit et al. 2018; Wisse et al. 2012). Both features could have influenced our results in terms of the optimal number of clusters. Due to the limited spatial resolution of MRI data, we may have missed differences in connectivity profiles of conflated subfields in the posterior hippocampus hence leading to a single tail cluster in the present study. Additionally, hippocampal gyrification, known as digitations, vary between individuals and have been previously discussed as a possible factor for inter- 
individual variability in the hippocampus (Chang et al. 2018; DeKraker et al. 2018; Ding and Van Hoesen 2015; Treit et al. 2018). How this affects cognition and psychopathology (Oppenheim et al. 1998) and whether different digitations have different connectivity profiles and hence could influence clustering pattern is still unclear. This question should be addressed in future studies with high spatial precision techniques. Overall, the maps and conceptual findings reported in the present study are useful for the specific mapping modality they have been derived from, that is, conventional field MRI in humans.

\section{Conclusions}

In the present study we established for the first time a robust and stable RSFC hippocampal parcellation by applying a combination of a model-free and a model-based denoising framework. By combining partitions based on spontaneous connectivity with partitions based on task-based connectivity, we built the first cross-modal generic hippocampal map at different levels of partition. Extensive behavioral profiling of the finest partition allowed inferences regarding the nature of information processing principles along the anterior-posterior axis in the hippocampus, beyond the concepts derived from psychological studies in specific fields. Importantly, while previous characterization of the anterior-posterior differentiation based on these concepts cannot be refuted and were partially supported, they could not account for the range of associations observed by our quantitative approaches. In turn, we proposed a self-world centric processing mode gradient along the anterior-posterior axis in humans, a databased hypothesis that should be further investigated with specific model-based approaches. Further functional decoding allowed us to speculate that the medial-lateral 
distinction represented an assimilating process for the lateral part integrating information across different systems. Importantly, our medial-lateral distinction for the first time evidenced that the anterior-posterior gradient is predominantly observed in the lateral part of the hippocampus and an independent mapping approach based on structural data (structural covariance) further evidenced a medial-lateral distinction. Finally, the pattern of separation revealed by structural covariance appeared as a hybridization of functional connectivity and microstructure hence bringing new light into this relatively understudied mapping modality and offering an alternative and potentially better partition for compression of structural data (cfr. (Varikuti et al. 2018)). All our uni-modal and cross-modal maps are available in the ANIMA database (http://anima.fz-juelich.de/) to support future hippocampal investigations of hippocampal function in healthy or pathological populations.

\section{Acknowledgments}

Our research was supported by the Deutsche Forschungsgemeinschaft (DFG, GE 2835/1-1, EI 816/4-1), the National Institute of Mental Health (R01-MH074457), the Helmholtz Portfolio Theme 'Supercomputing and Modelling for the Human Brain', and the European Union's Horizon 2020 Research and Innovation Programme under Grant Agreement No. 720270 (HBP SGA1) and No. 785907 (HBP SGA2). The authors also thank Dr. Nicola Palomero-Gallagher and Shahrzad Kharabian for fruitful discussions. 


\section{Supplemental methods}

\section{I.1 HCP functional data}

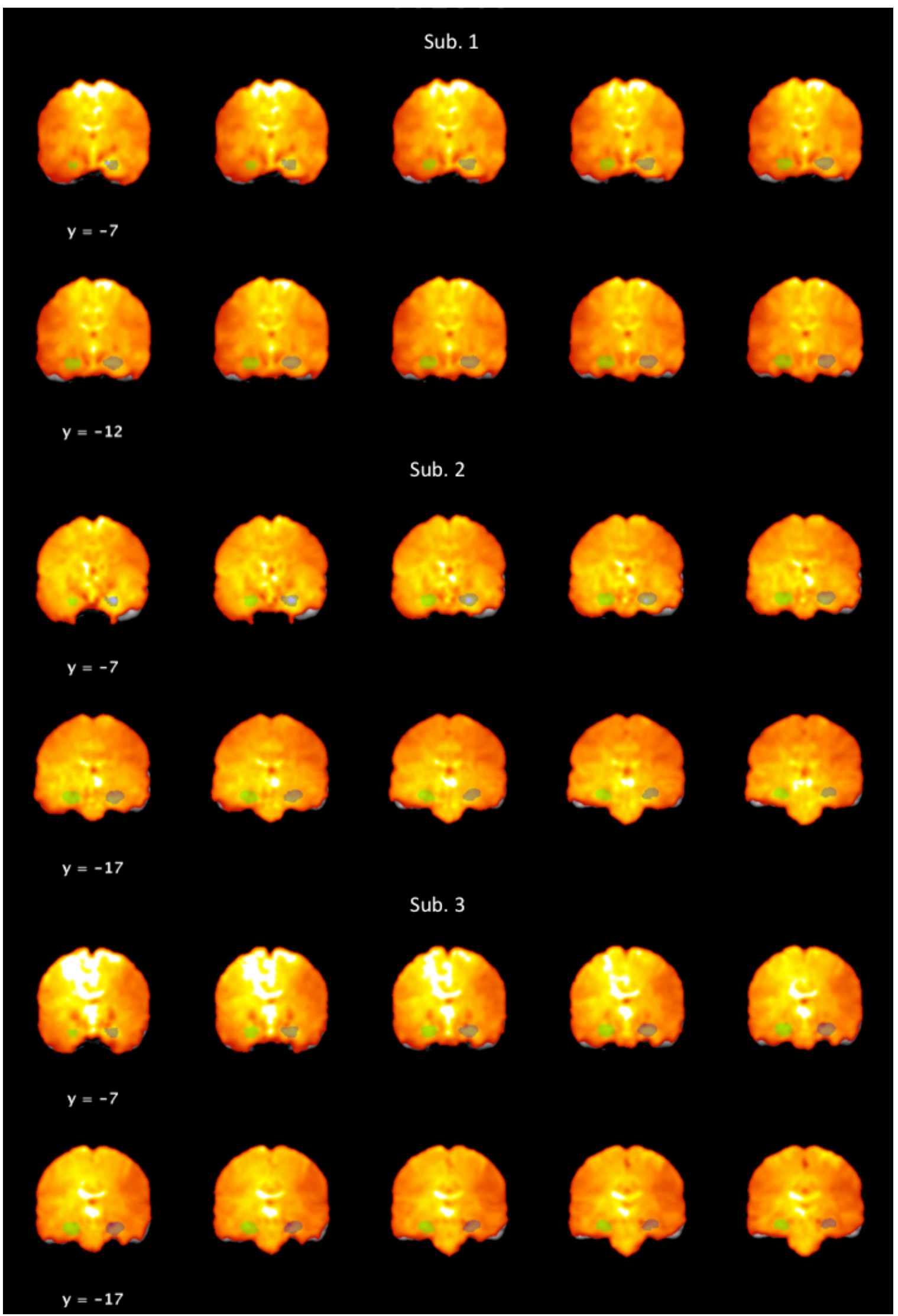


Sub. 4
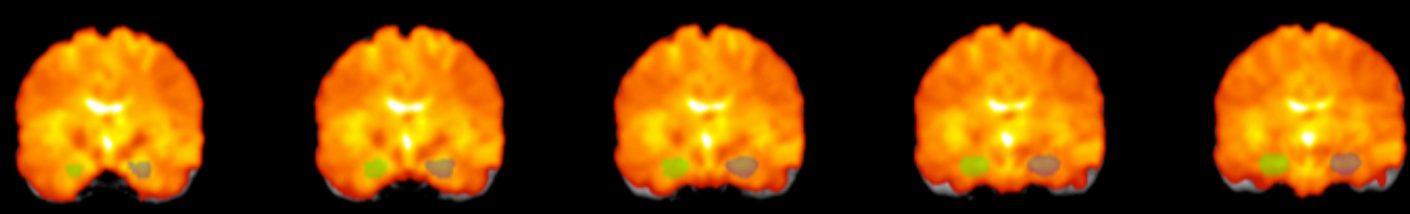

$y=-7$
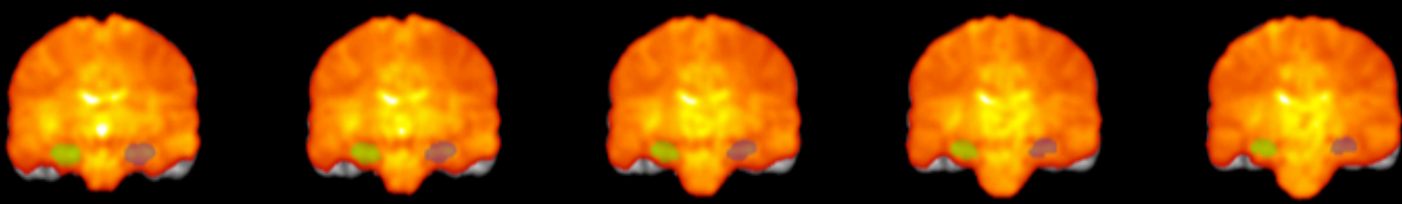

$y=-17$

Sub. 5
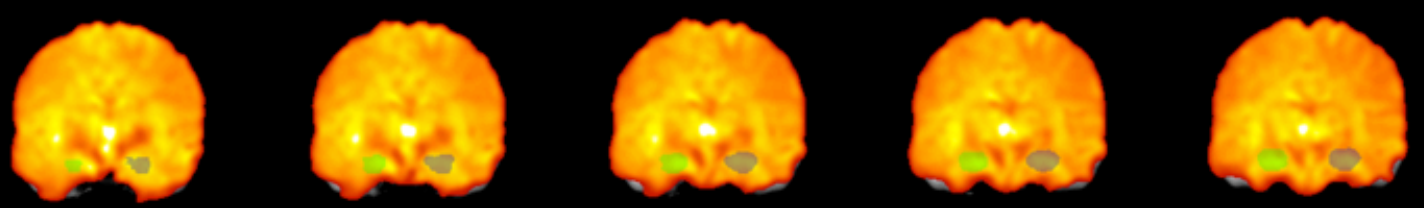

$y=-7$
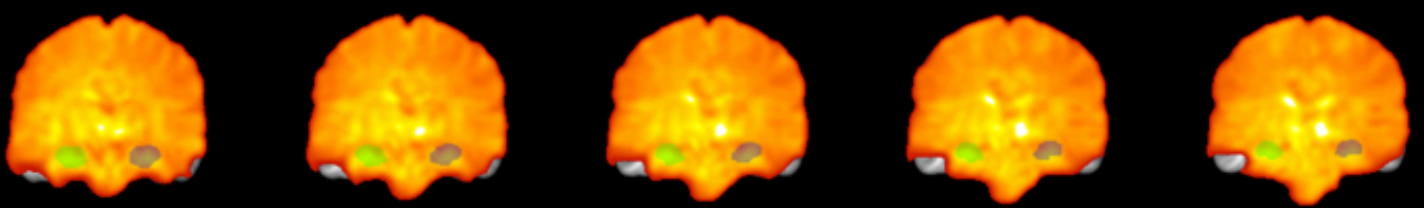

$y=-17$

Sub. 6
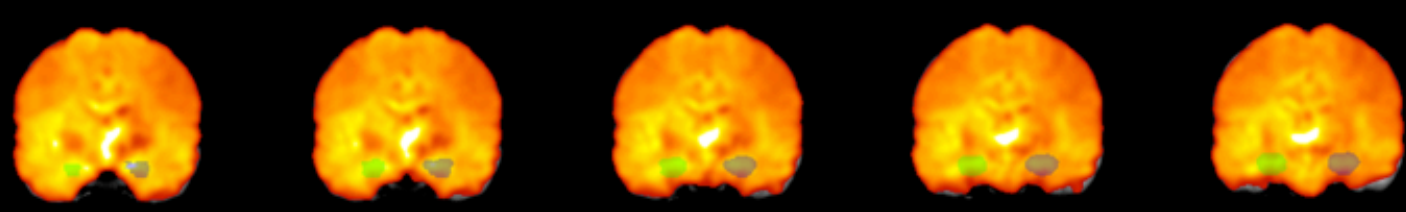

$y=-7$
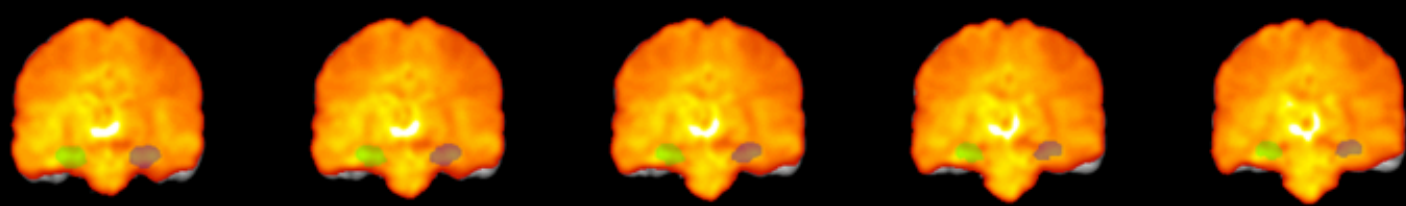

$y=-17$ 


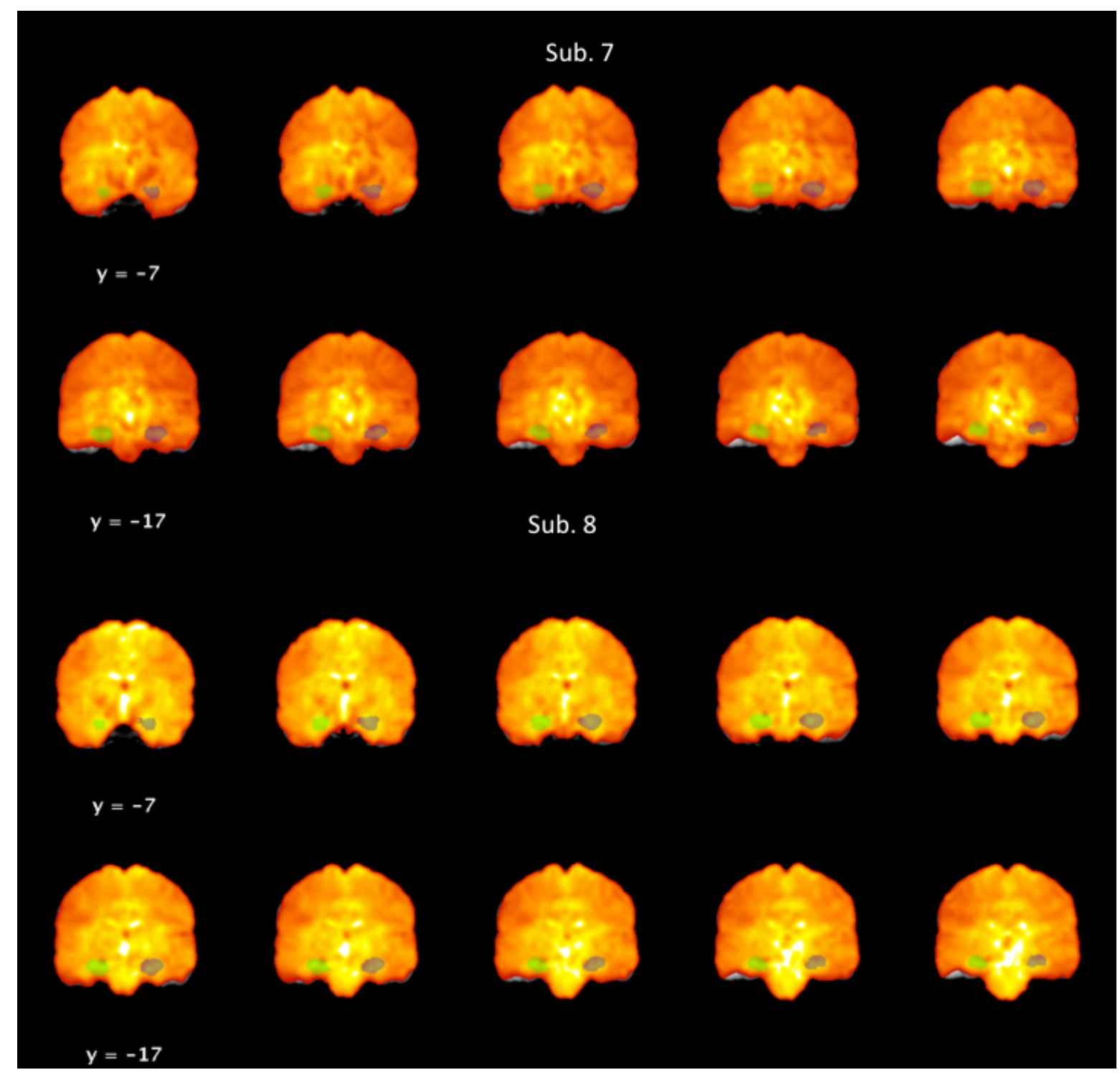




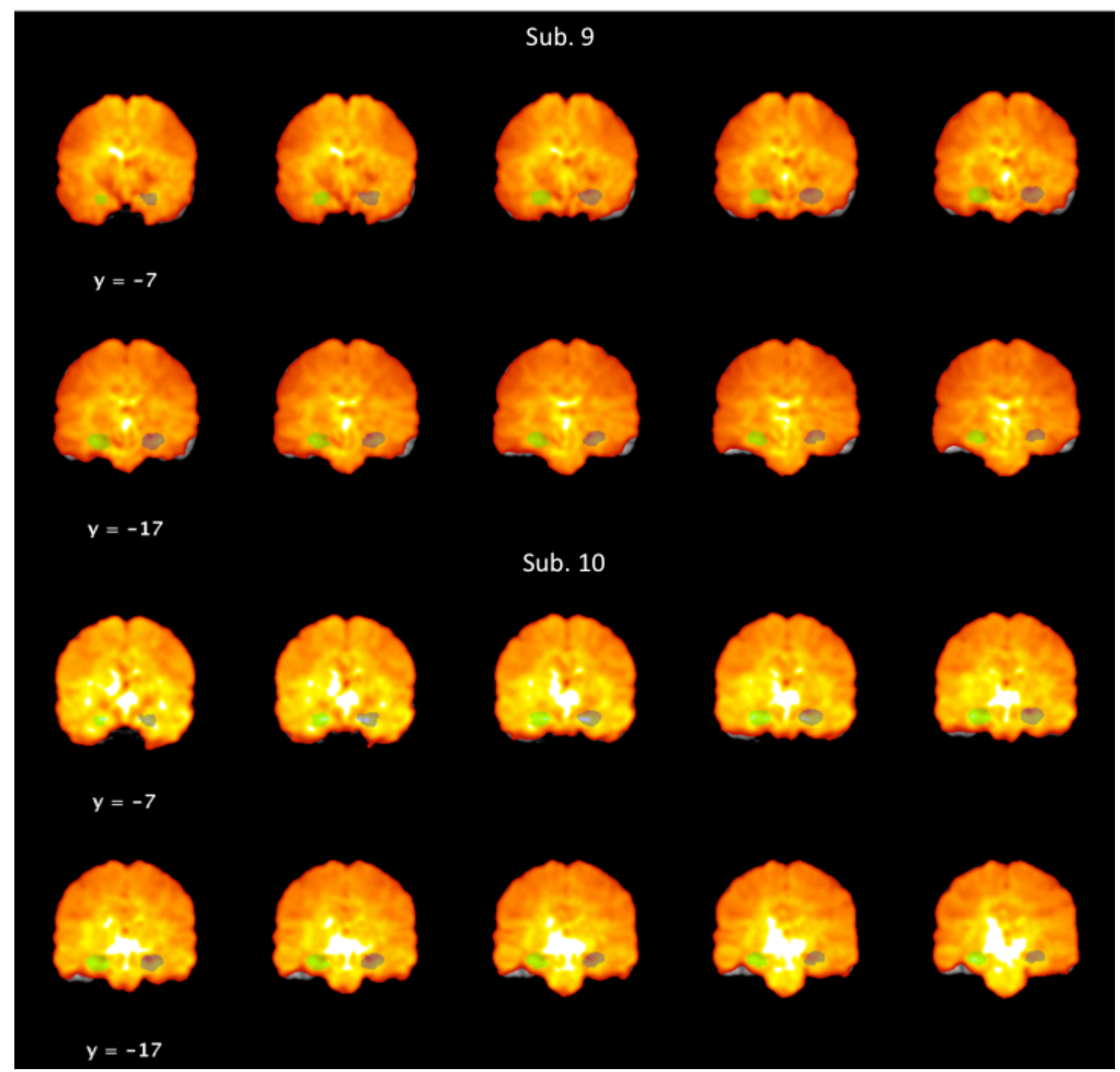

Figure 1. Resting-state functional signal in the hippocampus (green and blue ROI) of the HCP dataset on coronal slices.

\section{I.2 Circularity assumption}

A circularity limitation has been raised several times in previous studies parcellating brain regions based on MACM-CBP and characterizing these regions using the BrainMap database again. In those studies (Clos et al. 2013), MACM-CBP was performed in terms of whole-brain co-activation profiles of BrainMap to delineate subregions, and in the second step, the defined subregions were characterized in regard to associated behavioral concepts in this same database. Importantly, although both methods require BrainMap data, the underlying statistical computations and the type of data differ considerably. 
The initial analysis of identifying clusters for CBP is of exploratory nature and is based on an exploratory statistical framework (Eickhoff et al. 2015) using co-activations across studies and paradigms that were published. The next step of behavioral characterization is based on post-hoc inferential statistics (i.e. reverse inference P(Activation|Task) on associations between peaks and behavioral labels of the BrainMap database. Therefore we would like to emphasize that even though BrainMap data is used for both steps, MACM-CBP and behavioral profiling, the underlying statistical frameworks were different and were also applied on different types of data (whole brain co-activation maps vs behavioral domains) hence were less circular then assumed. Nevertheless, in the present study, we further addressed this potential circularity by first externally validate the pattern of MACM-CBP with RSFC-CBP and hence building a robust subdivision scheme. Furthermore, we validated externally the behavioral profiling of our clusters revealed by the analyses of the BrainMap database with an additional, independent database i.e. NeuroSynth. This procedure should have avoided circularity at any steps, parcellation and behavioral profiling.

I.3. Parcellation based on meta-analytic connectivity modeling (MACM-CBP) 


\section{Right hippocampus}

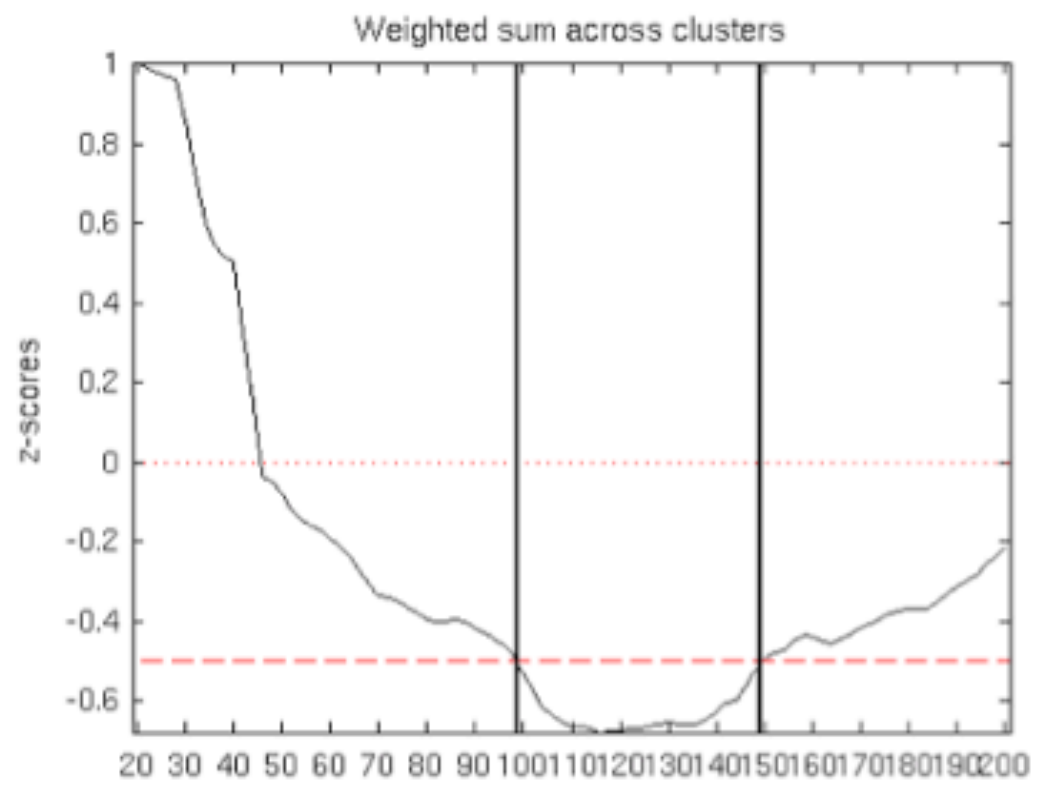

Left hippocampus

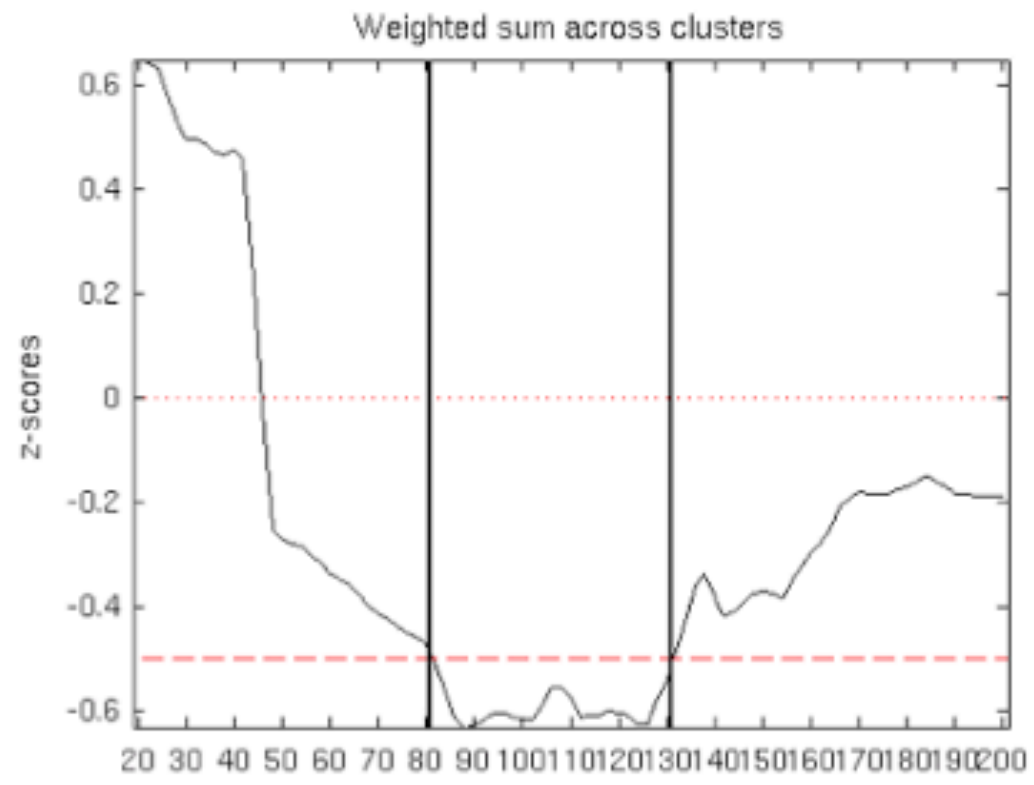

Figure 2. Filter range for MACM-CBP. Deviants and stability z-scores on median- filtered deviants. The vertical lines indicate the selected, most stable range of filter sizes (i.e., range with least deviants across k). Maximum z- score of median-filtered deviants. 


\section{I.4 Combination of denoising strategies}

In the history of fMRI and denoising the application of several subsequent cleanup strategies is not unusual. Different approaches were introduced in the past to remove different types of artifacts. The most common approach is to regress out confounders such as realignment parameters, tissue-specific signals (WM and/or CSF), global signals, and signals either from principal component or independent component analyses as stated in a recent report by (Satterthwaite et al. 2017).

Another recent work examined systematically what are the most common denoising approaches in use and how do they influence the resting state test-retest reliability (Varikuti et al. 2017). The literature survey of the authors revealed that combinations of different confounders are often performed. In particular, the most common strategies were global and tissue-class specific mean signal regression (either only WM/CSF or in combination with GSR) followed by principal component analysis in addition to GSR or with tissue specific confounders (Varikuti et al. 2017). ICA-based models like those underlying FIX, which is based on a machine learning approach, were less likely to be applied possibly because of its training requirements of the classifiers. Nevertheless, Griffanti et al. (2014) demonstrated the high accuracy performance (99\%) of these classifiers on HCP data to detect spatial artifacts, hence making FIX the first choice for such dataset. From a purely mathematical point of view, however, FIX was not designed to capture global or slow waves in the data, since it aims for an independent component separation (Griffanti et al. 2014) that does not apply to the interlocking global noise. In other words, it is highly sensitive for spatial artifacts in particular, but cannot capture global drifts. On the other hand, GSR is not selective at all but removes global waves with the negative effect of spatial distortions in functional connectivity, which can represent a major problem for parcellation work.

In line with this argument, Burgess et al. (2016), pointed out the limitation of using only FIX or GSR separately and combined both methods investigating its influence on motionartifacts. These were significantly reduced after both approaches were applied together and not separately (Burgess et al. 2016). In addition, Satterthwaite et al. (2017) pursued the same goal and confirmed that adding GSR to ICA-AROMA reduced motion artifacts significantly and provided the same performance as the classical denoising strategy with six realignment parameters, WM, CSF regression, and GSR applied jointly to the data. All their high performance models contained GSR. However, the use of GSR remains controversial because long-distance connections benefit from this approach, while short-distance connections do not as regionally specific artifacts cannot be captured by GSR (Satterthwaite et al. 2017). ICA-based denoising, on the other hand, did not show distance dependency, but left spurious stripes of motion artifacts unless GSR was added (Satterthwaite et al. 2017). In order to avoid distance dependency effects in our study (which could flawed the clustering), we included and tested another approach combining FIX with WM/CSF regression. We assumed that FIX would identify spatial artifacts and WM/CSF regression would compensate for physiological noise and, like GSR, all undulation influences of movement in the data. 
To demonstrate the effects of denoising we prepared some examples of grey ordinate plots for three subjects (Fig. 3) showing that the combination of model-based and model-free techniques do not distort the data. As expected, the plots show that the model-free strategies indeed additionally removed stripes present in the data that were not identified by FIX. Thus, in addition to the previous assumptions about the usefulness of a combined approach, our results also suggested that such a combination did not incorrectly distort the data. 


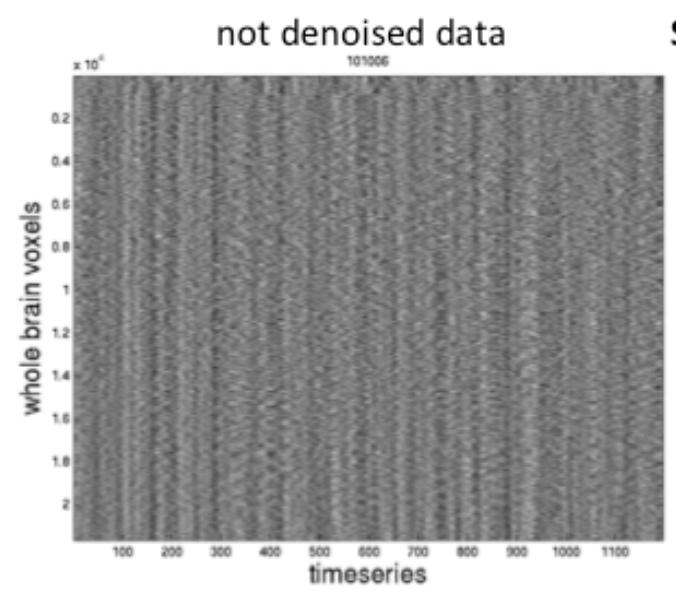

Sub. 1

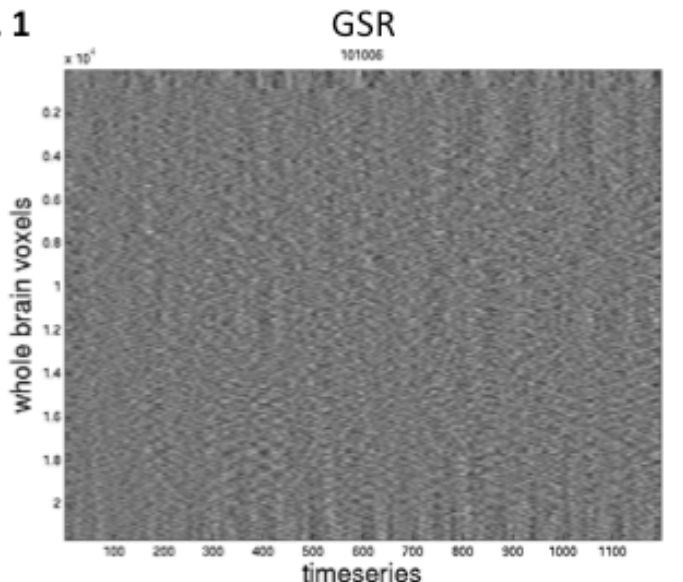

FIX

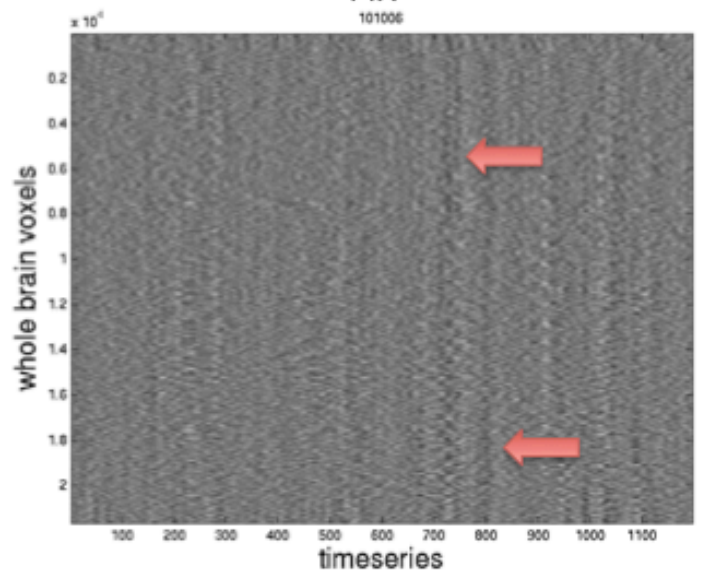

$\mathrm{FIX}+\mathrm{WM} / \mathrm{CSF}$
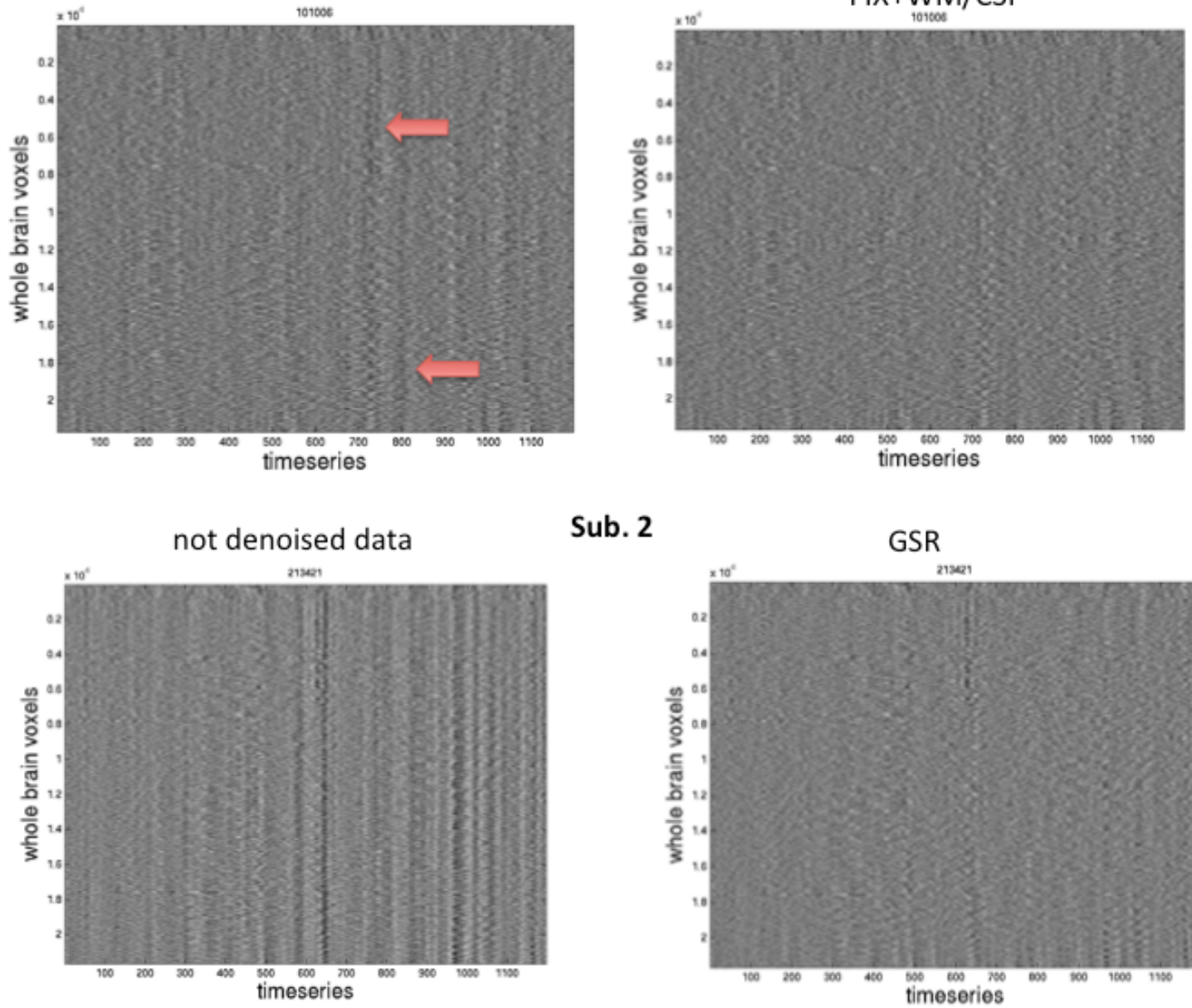

Sub. 2

GSR
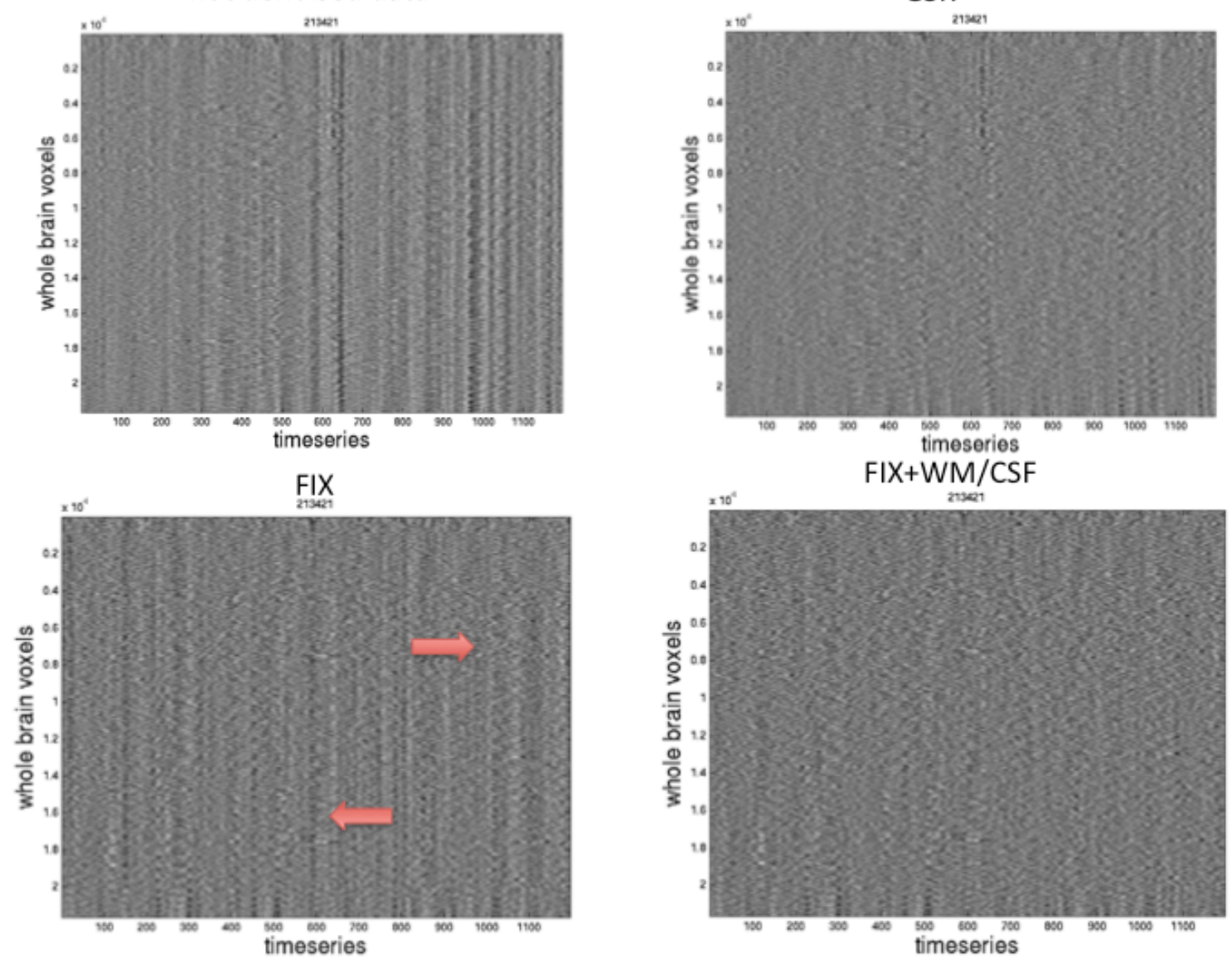


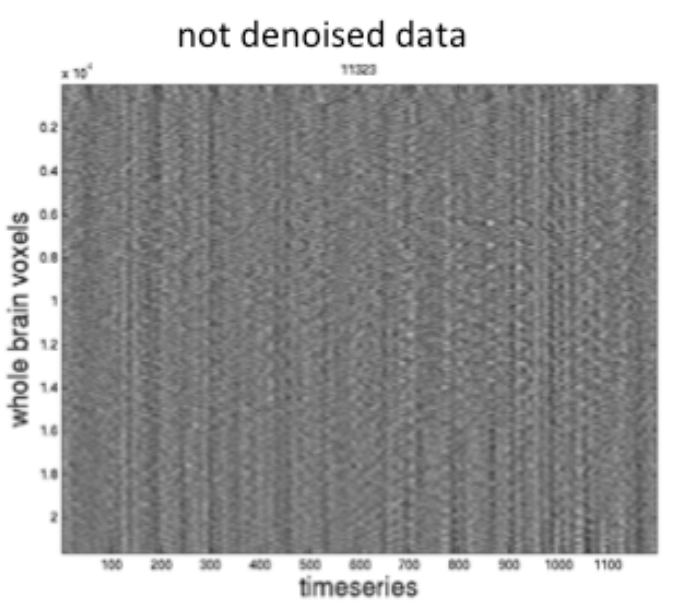

Sub. 3

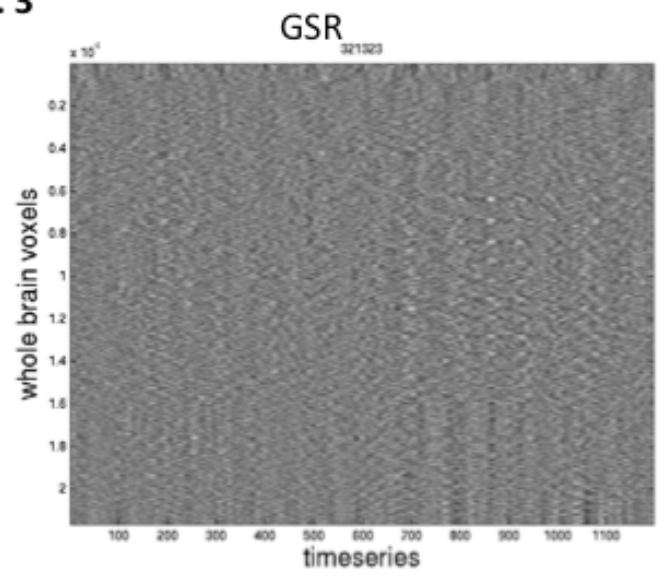

FIX

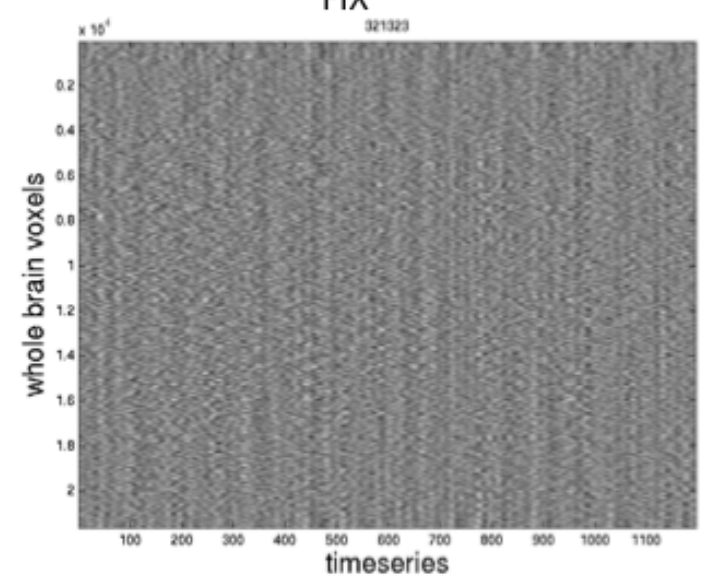

$\mathrm{FIX}+\mathrm{WM} / \mathrm{CSF}$

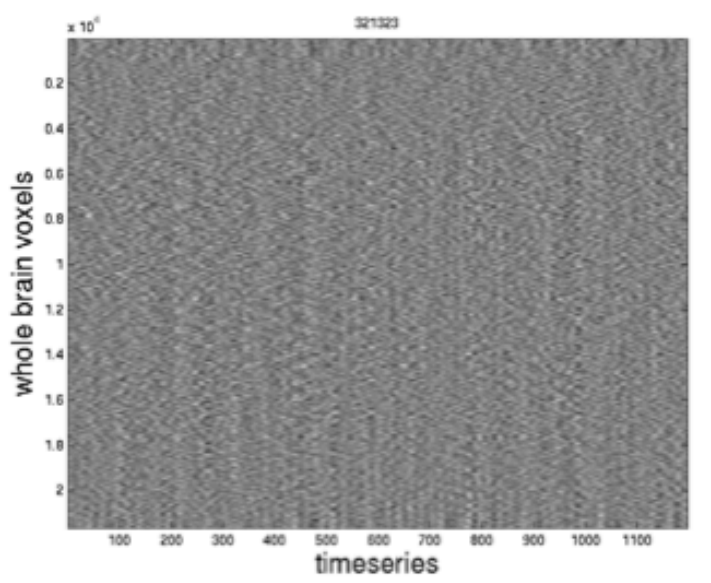

Figure 3. Influence of denoising on RS-fMRI signal. Grey ordinate plots for subject 1-3 for resting-state fMRI data either not denoised or denoised with different techniques demonstrating the removal of artifacts especially with FIX+WM/CSF achieving best results through removing stripes in the data as indicated with arrows.

\section{I.5 Measure of RSFC-CBP and SC-CBP}

For SC-CBP we had a matrix with 'participants $\mathrm{x}$ hippocampal grey matter values', which we correlated with another group-level matrix containing 'participants $x$ whole brain grey matter values' resulting in a group-level 'seed $x$ whole brain voxels' matrix. In contrast, for RSFC we correlated the seed voxels matrix across the time-series with whole brain voxels across time-series for every single subject (see Fig. 4). 


\section{RSFC-CBP}

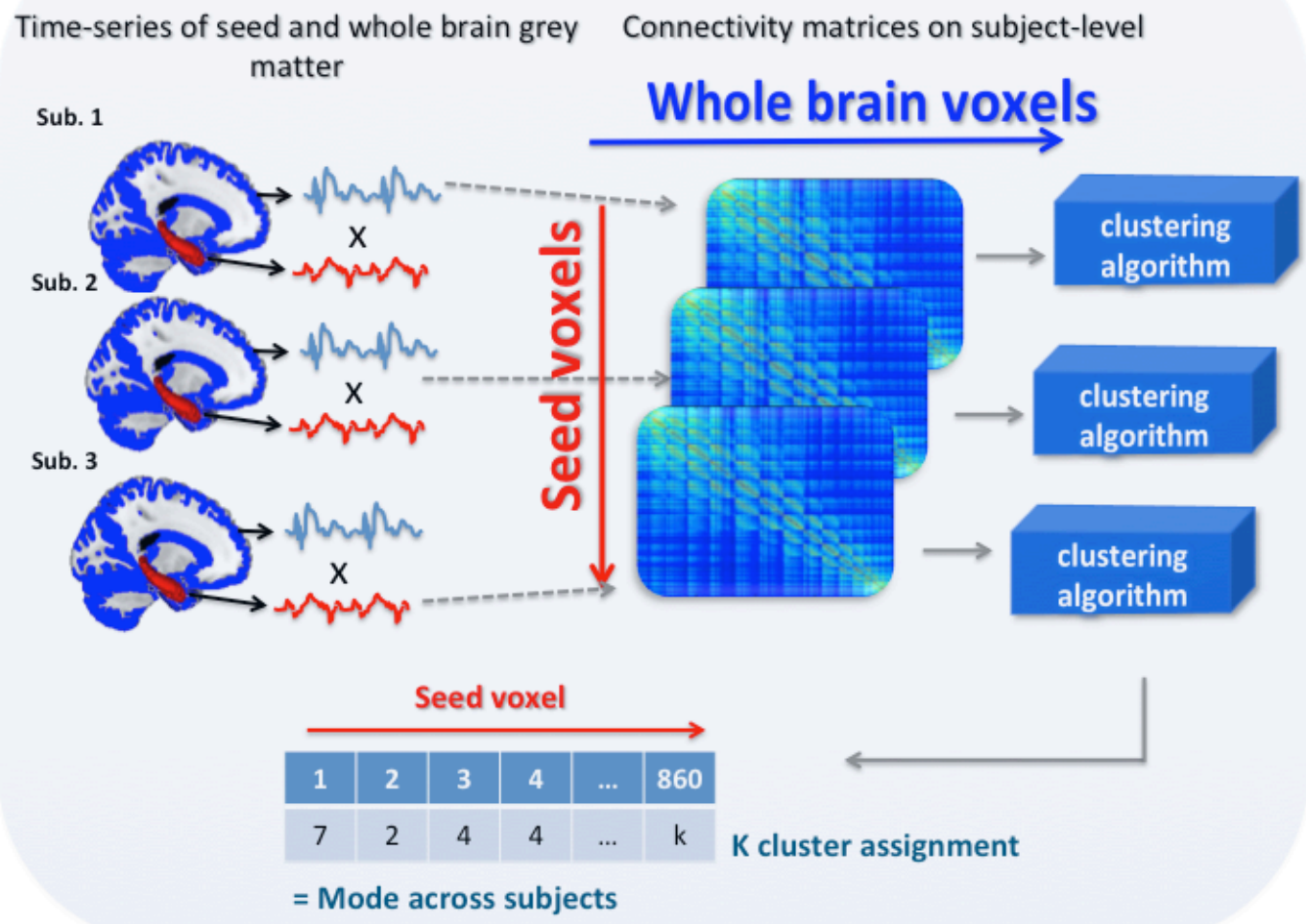

\section{SC-CBP}

Seed and whole brain grey matter throughout the sample

Connectivity matrices on group-level Sub. 1

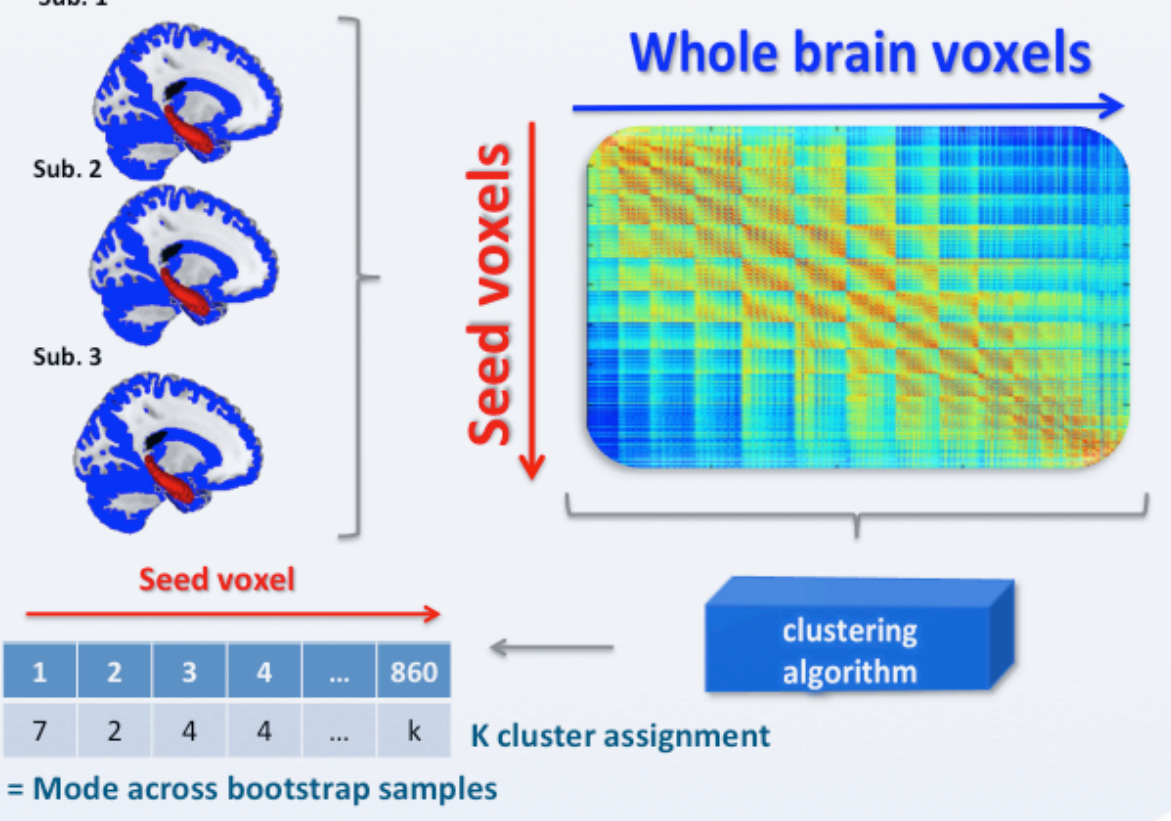

Figure 4. Connectivity input of RSFC-CBP and SC-CBP for the clustering algorithm and its output vector of seed voxels assigned to clusters. 


\section{I.6 Adjusted Rand Index}

The adjusted rand index reflects the dissimilarity between partitions based on the number of pairs of elements (here voxels) that are either clustered together or separately in partitions. For example, if two given, voxel A and voxel B, are clustered together in partition $X$ and also in partition $Y$, these two partitions agrees for this pair of voxels $A-B$. In contrast, if voxel $A$ and voxel $B$ are clustered together in partition $X$, while voxel $A$ and voxel $B$ are assigned to different clusters in partition $Y$, the two partitions, $X$ and $Y$ disagree for this pair of voxels A-B. Taking into account the total number of pairs, we can make a statement about how many pairs of voxels in both parts agree, and how many do not. Therefore in the numerator the agreement between clusterings is expressed (pairs of voxels that are found in both clusterings) whereas in the dominator the total number of pairs is specified.

In order to compare two partitions/clusterings we used a matrix containing for each hippocampal voxel the cluster number to which it was assigned across participants (RSFC, SC) or filter sizes (MACM). For stability measures in RSFC, we divided the matrix randomly into two halves based on the number of participants. Each half contained all hippocampal voxels assigned to clusters within one half of the participants. We computed the mode of cluster assignment across this half, resulting in a vector containing only the hippocampal voxels and their most frequent cluster labels for the specific half. We compared the two vectors resulting from the two halves with each other using the ARI as shown in Fig. 5. For validity measurements, we used the same procedure except that the entire matrix with all bootstrapped participants (or filter sizes) was used to generate the hippocampal vectors (see Fig. 5). 


\section{ARI measure within modality RSFC-CBP}
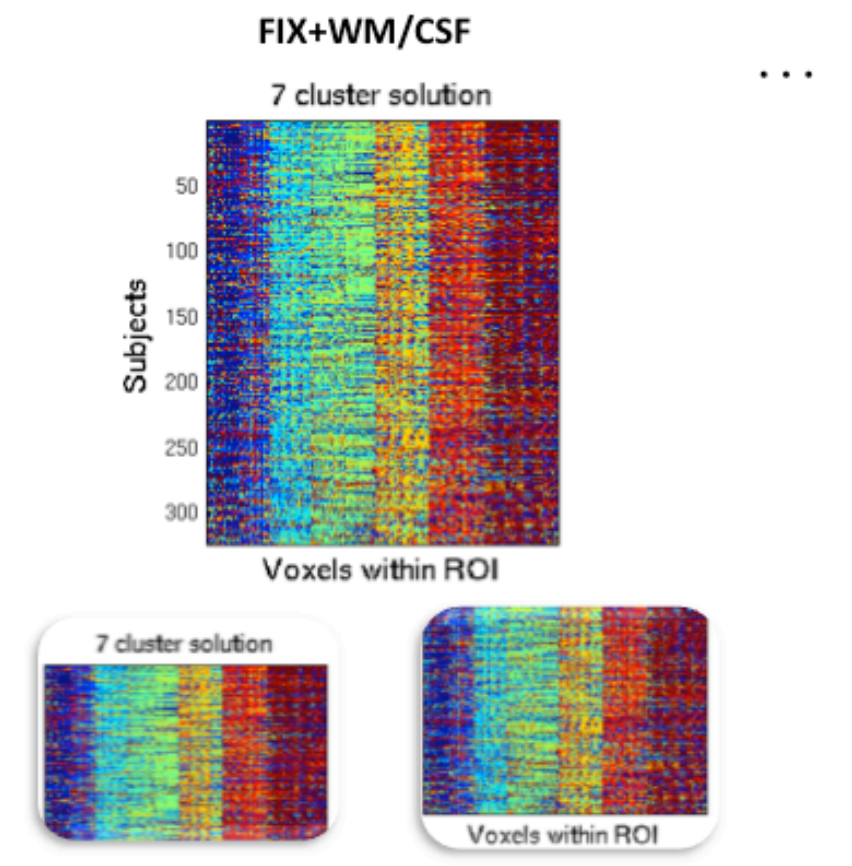

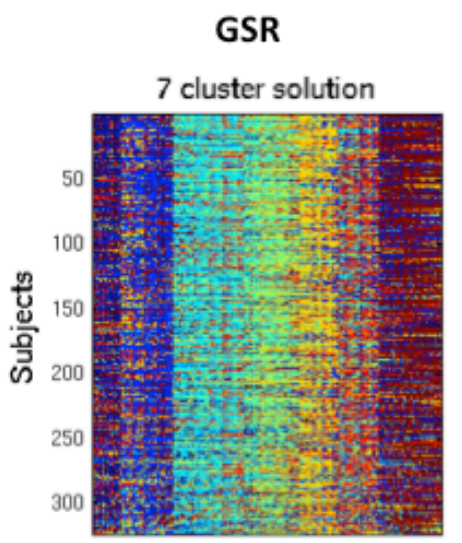

Voxels within ROI
1. split half

Seed voxel

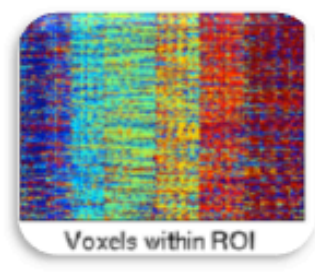

2. split half

Seed voxel

\begin{tabular}{|c|c|c|c|c|c|c|c|c|c|c|c|c|c|}
\hline 1 & 2 & 3 & 4 & $\ldots$ & 860 & ARI & 1 & 2 & 3 & 4 & $\ldots$ & 860 & \\
\hline 1 & 2 & 4 & 7 & $\ldots$ & $\mathrm{k}$ & $\longleftrightarrow$ & 7 & 2 & 4 & 4 & $\ldots$ & k & $\mathrm{K}$ cluster number \\
\hline
\end{tabular}

\section{ARI measure between modalities}

RSFC (FIX+WM/CSF)

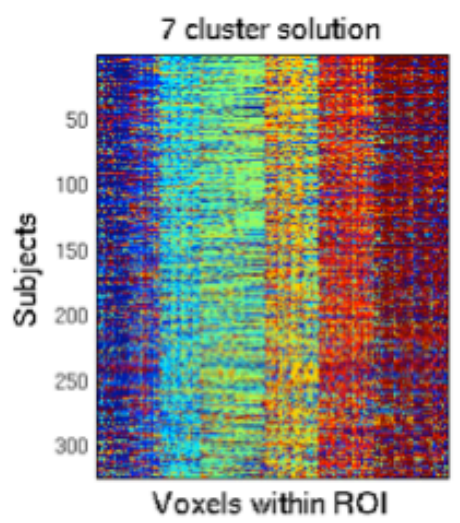

Seed voxel

\begin{tabular}{|c|c|c|c|c|c|}
\hline 1 & 2 & 3 & 4 & $\ldots$ & 860 \\
\hline 1 & 2 & 4 & 7 & $\ldots$ & $k$ \\
\hline
\end{tabular}

$=$ Mode across subjects
MACM

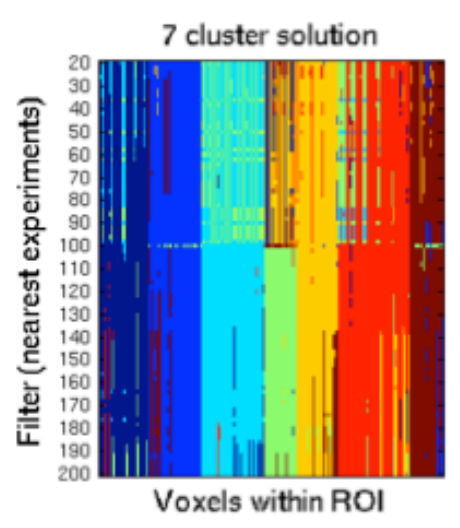

ARI

Seed voxel

\begin{tabular}{|c|c|c|c|c|c|}
\hline 1 & 2 & 3 & 4 & $\ldots$ & 860 \\
\hline 4 & 2 & 6 & 7 & $\ldots$ & $k$ \\
\hline
\end{tabular}

Figure 5. ARI measure within and between modalities. 


\section{I.7 Cluster characterization with BrainMap and NeuroSynth databases}

For NeuroSynth we used the following centroid coordinates of each cluster in MNI152 space in order to associate specific behavioral profiles to the partitions.

Consensus cluster

$\mathbf{X}$

$\mathbf{y}$

$\mathbf{Z}$

\section{Right hippocampus}

Anterior rostral cluster

$22-10 \quad-24$

Anterior lateral cluster

31

$-16 \quad-20$

Anterior medial cluster

$21 \quad-17 \quad-17$

Intermediate lateral cluster

$\begin{array}{lll}33 & -26 & -12\end{array}$

Intermediate medial 2 cluster

$26-22 \quad-16$

Intermediate medial 1 cluster

$24-31 \quad-9$

Posterior cluster

26

$-37 \quad-2$

\section{Left hippocampus}

Anterior rostral cluster

$\begin{array}{lll}-23 & -11 & -24\end{array}$

Anterior lateral cluster

$\begin{array}{lll}-31 & -15 & -21\end{array}$

Anterior medial cluster

$\begin{array}{lll}-24 & -20 & -18\end{array}$

Intermediate lateral 2 cluster

$\begin{array}{lll}-33 & -24 & -14\end{array}$

Intermediate lateral 1 cluster

$\begin{array}{lll}-31 & -35 & -7\end{array}$

Intermediate medial cluster

$\begin{array}{lll}-26 & -30 & -9\end{array}$

Posterior cluster

$-20$

$-35 \quad 1$

Table 1. Coordinates of the generic hippocampal map for NeuroSynth characterization

II. Supplemental results

\section{II.1 Extending decision criteria for optimal cluster number}

Throughout our work two major criteria were responsible for our decision for the optimal number of partitions: stability measured with split-half cross-validation and biological meaningfulness estimated by bootstrap resampling across MRI modalities and compared with the ARI. For the sake of completeness we also examined an index of data 
representation within each connectivity modality. We here used Variation of Information (VI) as suggested by Kelly et al. (2012). This metric measures the distance between two clusterings and indicates how much information is lost or captured when switching from one cluster solution to another clustering (Meilă 2007). Accordingly, a cluster solution is considered as optimal when it does not show an increase of VI compared to other cluster solutions so that low values show optimal solutions in relation to previous or subsequent cluster solutions. We here examined this metric for cluster solutions ranging from two to ten cluster solutions. Overall, VI further supported the 7 cluster solution as an optimal level of compression for both functional connectivity measures as summarized in Fig. 6.

In particular, for RSFC, VI tends to decrease from the 6-cluster to the 7-cluster solution, but either to remain stable (right hippocampus) or to increase (left hippocampus) from the 7-cluster solution to the 8-cluster solution. For MACM, VI showed a clear decrease from the 6-cluster solution to the 7-cluster solution, but an increase from the latter to the next solution in the left hippocampus. Somewhat surprisingly, the pattern was different in the right hippocampus with VI showing a progressive increase from the 5cluster solution up to the 9 cluster solution, thus a pattern that differs with what is observed with RSFC. We could therefore assume that this difference is related to technical factors (such as overall more variance in activation peaks reported in the left hippocampus), despite the influence of a biological factor for this observation cannot be ruled out. Although SC parcellation was not incorporated in our consensus functional parcellation (based on RSFC and MACM), for the sake of completeness, we also examined VI in clustering based on SC. This reveals that VI decreases from a 5-cluster solution to a 6-cluster solution, but then shows a "plateau" until the 10 cluster solution for which it increases when compared to the 9 cluster-solution in the right hemisphere. In contrast, on the left hippocampus, VI clearly reaches an optimum at a 7-cluster level. Thus, in sum, the VI criterion supported the 7 cluster solution as an optimal representation of connectivity variance as estimated by different features (MACM, RSFC, SC) in particular in the left hippocampus. We noted that the right hippocampus showed a slightly more complex pattern with differences across features; nevertheless, hemispheric differences are beyond the scope of the current study. Overall, both the investigation of VI across cluster solution and our previous investigation of stability support a 7-cluster subdivision as an optimal compression of MRI functional connectivity features in the hippocampus. 

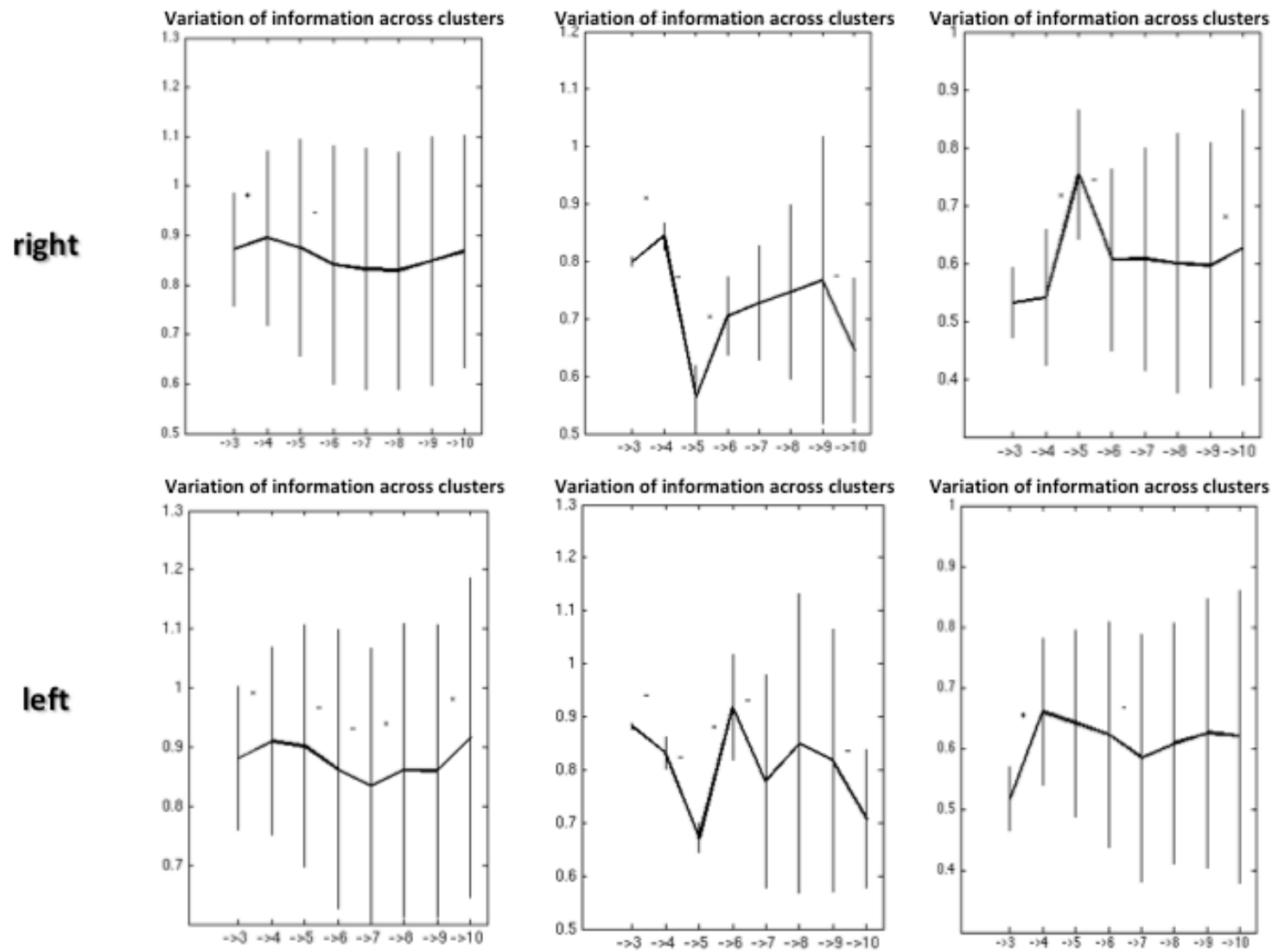

Figure 6. Variation of information across modalities and hemispheres supported cluster solution 7 for RSFC and SC-CBP, and 5 for MACM-CBP.

\section{II.2 Functional consensus map}

In order to be able to establish a consensus map that was generally representing functional architecture of the hippocampus we decided to merge the 10000 generated bootstrap samples of MACM and RSFC as these two modalities exposed the highest convergence across modalities visually and in regard to the ARI index as represented in the following table 2. SC, on the other hand, had a hybrid-like nature with a functional head division that was similar to MACM and RSFC and a body and tail partitioning reminding of the cytoarchitectonic subfields for which it was excluded from the crossmodal map. 


\begin{tabular}{|c|c|c|}
\hline \multirow[t]{2}{*}{ right } & \multicolumn{2}{|c|}{ cluster solution 3} \\
\hline & MACM-CBP & SC-CBP \\
\hline RSFC-CBP & 0.4645 & 0.3891 \\
\hline \multirow[t]{2}{*}{ МАСМ-СВР } & 1 & 0.5260 \\
\hline & \multicolumn{2}{|c|}{ cluster solution 5} \\
\hline RSFC-CBP & 0.6514 & 0.3984 \\
\hline \multirow[t]{2}{*}{ MACM-CВP } & 1 & 0.3686 \\
\hline & \multicolumn{2}{|c|}{ cluster solution 7} \\
\hline RSFC-CBP & 0.4641 & 0.2937 \\
\hline МАСМ-СВР & 1 & 0.3361 \\
\hline left & \multicolumn{2}{|c|}{ cluster solution 3} \\
\hline RSFC-CBP & 0.4963 & 0.3751 \\
\hline \multirow[t]{2}{*}{ МACM-СВР } & 1 & 0.4667 \\
\hline & \multicolumn{2}{|c|}{ cluster solution 5} \\
\hline RSFC-CBP & 0.4642 & 0.3759 \\
\hline \multirow[t]{2}{*}{ МАСМ-СВР } & 1 & 0.4838 \\
\hline & \multicolumn{2}{|c|}{ cluster solution 7} \\
\hline RSFC-CBP & 0.5116 & 0.3715 \\
\hline МACM-CBP & 1 & 0.3592 \\
\hline
\end{tabular}

Table 2. Clustering similarity (measured with ARI index) across CBP modalities.

II.3 Clustering based on unsmoothed data 


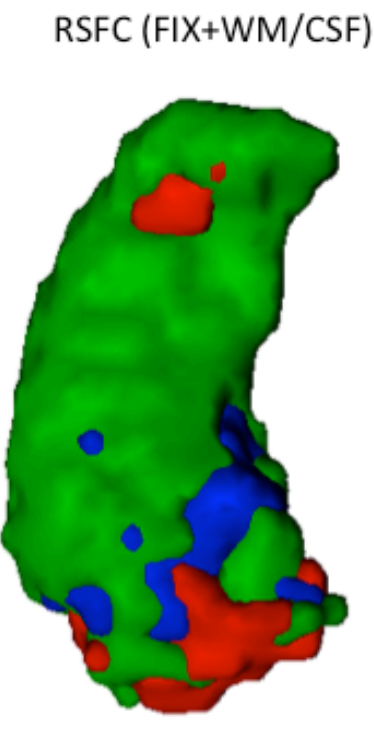

RSFC (FIX+WM/CSF)

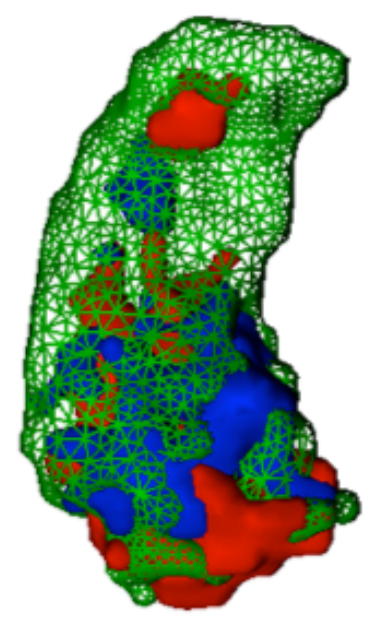

SC

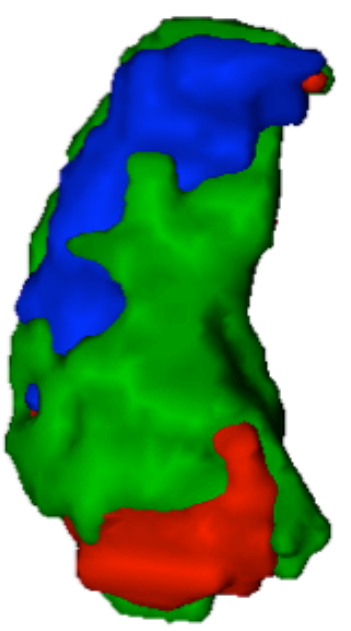

SC

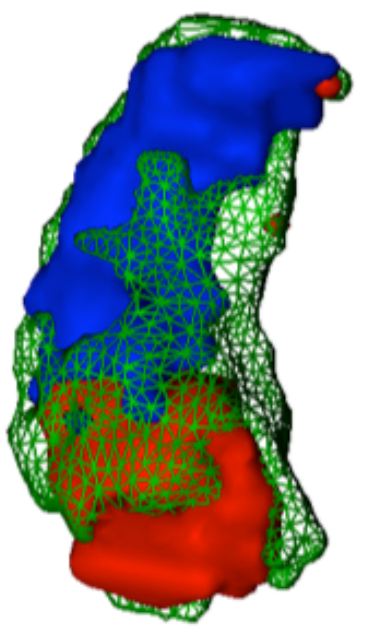

RSFC (FIX+WM/CSF)
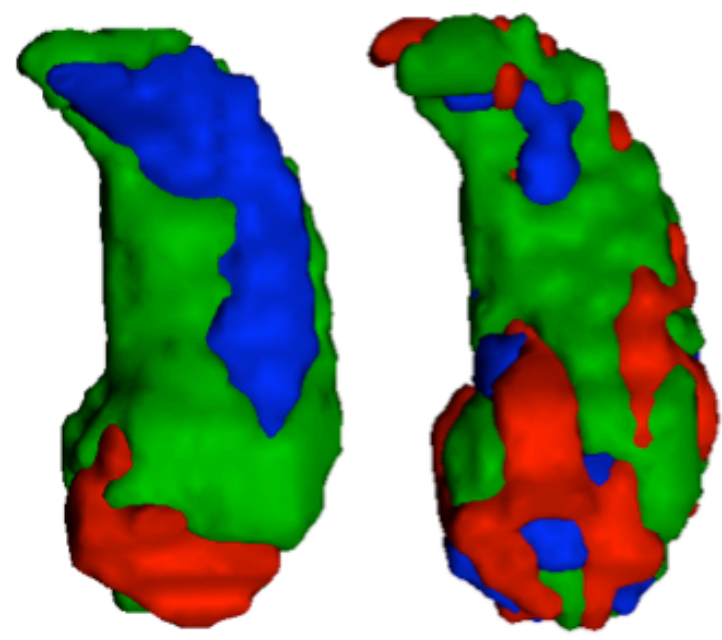

RSFC (FIX+WM/CSF)
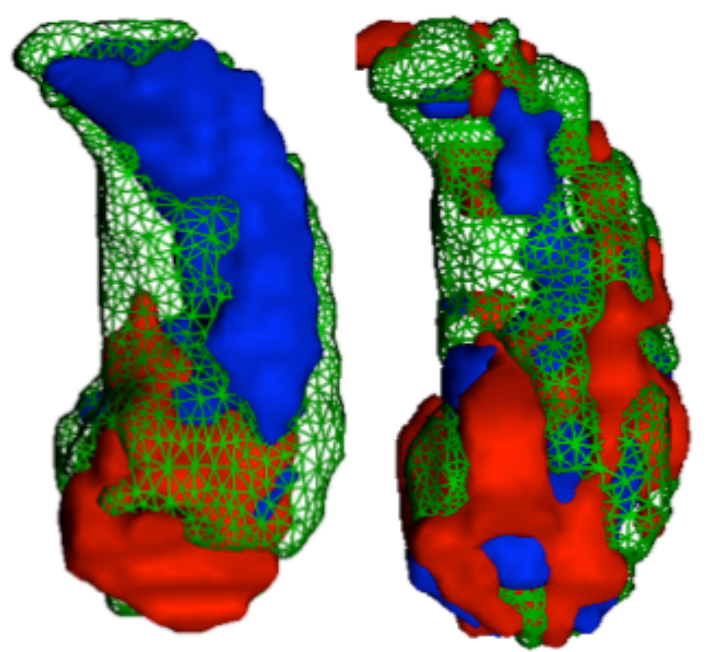

Figure 7. Unsmoothed hippocampi for RSFC and SC modality. Fragmented clustering still indicates the already known pattern from smoothed data, with RSFC showing an anterior-posterior differentiation and SC a cytoarchitectonic-like pattern. Lower row shows green transparent clusters in order to illustrate the underlying organization. 


\section{References}

Adler DH, Pluta J, Kadivar S, Craige C, Gee JC, Avants BB, Yushkevich PA. 2014. Histologyderived volumetric annotation of the human hippocampal subfields in postmortem mri. NeuroImage. 84:505-523.

Adler DH, Wisse LEM, Ittyerah R, Pluta JB, Ding SL, Xie L, Wang J, Kadivar S, Robinson JL, Schuck $\mathrm{T}$ et al. 2018. Characterizing the human hippocampus in aging and alzheimer's disease using a computational atlas derived from ex vivo mri and histology. Proceedings of the National Academy of Sciences of the United States of America. 115(16):4252-4257.

Adnan A, Barnett A, Moayedi M, McCormick C, Cohn M, McAndrews MP. 2016. Distinct hippocampal functional networks revealed by tractography-based parcellation. Brain structure \& function. 221(6):2999-3012.

Alexander-Bloch A, Raznahan A, Bullmore E, Giedd J. 2013. The convergence of maturational change and structural covariance in human cortical networks. The Journal of neuroscience : the official journal of the Society for Neuroscience. 33(7):2889-2899.

Amunts K, Kedo 0, Kindler M, Pieperhoff P, Mohlberg H, Shah NJ, Habel U, Schneider F, Zilles K. 2005. Cytoarchitectonic mapping of the human amygdala, hippocampal region and entorhinal cortex: Intersubject variability and probability maps. Anatomy and embryology. 210(5-6):343-352.

Anderson JS, Druzgal TJ, Lopez-Larson M, Jeong EK, Desai K, Yurgelun-Todd D. 2011. Network anticorrelations, global regression, and phase-shifted soft tissue correction. Human brain mapping. 32(6):919-934.

Arslan S, Ktena SI, Makropoulos A, Robinson EC, Rueckert D, Parisot S. 2018. Human brain mapping: A systematic comparison of parcellation methods for the human cerebral cortex. NeuroImage. 170:5-30.

Ashburner J, Friston KJ. 2005. Unified segmentation. NeuroImage. 26(3):839-851.

Bakker A, Kirwan CB, Miller M, Stark CE. 2008. Pattern separation in the human hippocampal ca3 and dentate gyrus. Science (New York, NY). 319(5870):16401642.

Beckmann CF, Smith SM. 2004. Probabilistic independent component analysis for functional magnetic resonance imaging. IEEE transactions on medical imaging. 23(2):137-152.

Behrens TE, Johansen-Berg H, Woolrich MW, Smith SM, Wheeler-Kingshott CA, Boulby PA, Barker GJ, Sillery EL, Sheehan K, Ciccarelli O et al. 2003. Non-invasive mapping of connections between human thalamus and cortex using diffusion imaging. Nature neuroscience. 6(7):750-757.

Bellec P, Rosa-Neto P, Lyttelton OC, Benali H, Evans AC. 2010. Multi-level bootstrap analysis of stable clusters in resting-state fmri. NeuroImage. 51(3):1126-1139.

Bianciardi M, Fukunaga M, van Gelderen P, Horovitz SG, de Zwart JA, Shmueli K, Duyn JH. 2009. Sources of functional magnetic resonance imaging signal fluctuations in the human brain at rest: A 7 t study. Magnetic resonance imaging. 27(8):1019-1029.

Birn RM, Diamond JB, Smith MA, Bandettini PA. 2006. Separating respiratory-variationrelated fluctuations from neuronal-activity-related fluctuations in fmri. NeuroImage. 31(4):1536-1548.

Biswal B, Yetkin FZ, Haughton VM, Hyde JS. 1995. Functional connectivity in the motor cortex of resting human brain using echo-planar mri. Magnetic resonance in medicine. 34(4):537-541.

Buckner RL, Krienen FM, Yeo BT. 2013. Opportunities and limitations of intrinsic functional connectivity mri. Nature neuroscience. 16(7):832-837. 
Burgess GC, Kandala S, Nolan D, Laumann TO, Power JD, Adeyemo B, Harms MP, Petersen SE, Barch DM. 2016. Evaluation of denoising strategies to address motion-correlated artifacts in resting-state functional magnetic resonance imaging data from the human connectome project. Brain connectivity. 6(9):669680.

Caballero-Gaudes C, Reynolds RC. 2017. Methods for cleaning the bold fmri signal. NeuroImage. 154:128-149.

Chang C, Huang C, Zhou N, Li SX, Ver Hoef L, Gao Y. 2018. The bumps under the hippocampus. Human brain mapping. 39(1):472-490.

Chase HW, Clos M, Dibble S, Fox P, Grace AA, Phillips ML, Eickhoff SB. 2015. Evidence for an anterior-posterior differentiation in the human hippocampal formation revealed by meta-analytic parcellation of fmri coordinate maps: Focus on the subiculum. NeuroImage. 113:44-60.

Clos M, Amunts K, Laird AR, Fox PT, Eickhoff SB. 2013. Tackling the multifunctional nature of broca's region meta-analytically: Co-activation-based parcellation of area 44. NeuroImage. 83:174-188.

Clos M, Rottschy C, Laird AR, Fox PT, Eickhoff SB. 2014. Comparison of structural covariance with functional connectivity approaches exemplified by an investigation of the left anterior insula. Neurolmage. 99:269-280.

Cole DM, Smith SM, Beckmann CF. 2010. Advances and pitfalls in the analysis and interpretation of resting-state fmri data. Frontiers in systems neuroscience. 4:8.

Colombo M, Fernandez T, Nakamura K, Gross CG. 1998. Functional differentiation along the anterior-posterior axis of the hippocampus in monkeys. J Neurophysiol. 80(2):1002-1005.

Dagli MS, Ingeholm JE, Haxby JV. 1999. Localization of cardiac-induced signal change in fmri. NeuroImage. 9(4):407-415.

de Flores R, La Joie R, Chetelat G. 2015. Structural imaging of hippocampal subfields in healthy aging and alzheimer's disease. Neuroscience. 309:29-50.

de Hoz L, Knox J, Morris RG. 2003. Longitudinal axis of the hippocampus: Both septal and temporal poles of the hippocampus support water maze spatial learning depending on the training protocol. Hippocampus. 13(5):587-603.

de Munck JC, Goncalves SI, Faes TJ, Kuijer JP, Pouwels PJ, Heethaar RM, Lopes da Silva FH. 2008. A study of the brain's resting state based on alpha band power, heart rate and fmri. NeuroImage. 42(1):112-121.

DeKraker J, Ferko KM, Lau JC, Kohler S, Khan AR. 2018. Unfolding the hippocampus: An intrinsic coordinate system for subfield segmentations and quantitative mapping. NeuroImage. 167:408-418.

Desikan RS, Segonne F, Fischl B, Quinn BT, Dickerson BC, Blacker D, Buckner RL, Dale AM, Maguire RP, Hyman BT et al. 2006. An automated labeling system for subdividing the human cerebral cortex on mri scans into gyral based regions of interest. NeuroImage. 31(3):968-980.

Desjardins AE, Kiehl KA, Liddle PF. 2001. Removal of confounding effects of global signal in functional mri analyses. NeuroImage. 13(4):751-758.

Deuker L, Doeller CF, Fell J, Axmacher N. 2014. Human neuroimaging studies on the hippocampal ca3 region - integrating evidence for pattern separation and completion. Frontiers in cellular neuroscience. 8:64.

Dimsdale-Zucker HR, Ritchey M, Ekstrom AD, Yonelinas AP, Ranganath C. 2018. Ca1 and ca3 differentially support spontaneous retrieval of episodic contexts within human hippocampal subfields. Nature communications. 9(1):294. 
Ding SL, Van Hoesen GW. 2015. Organization and detailed parcellation of human hippocampal head and body regions based on a combined analysis of cyto- and chemoarchitecture. The Journal of comparative neurology. 523(15):2233-2253.

Dong HW, Swanson LW, Chen L, Fanselow MS, Toga AW. 2009. Genomic-anatomic evidence for distinct functional domains in hippocampal field ca1. Proceedings of the National Academy of Sciences of the United States of America. 106(28):11794-11799.

Efron B. 1979. Bootstrap methods: Another look at the jackknife. Ann Statist. 7(1):1-26.

Eickhoff SB, Bzdok D, Laird AR, Kurth F, Fox PT. 2012. Activation likelihood estimation meta-analysis revisited. NeuroImage. 59(3):2349-2361.

Eickhoff SB, Stephan KE, Mohlberg H, Grefkes C, Fink GR, Amunts K, Zilles K. 2005. A new spm toolbox for combining probabilistic cytoarchitectonic maps and functional imaging data. NeuroImage. 25(4):1325-1335.

Eickhoff SB, Thirion B, Varoquaux G, Bzdok D. 2015. Connectivity-based parcellation: Critique and implications. Human brain mapping. 36(12):4771-4792.

Eickhoff SB, Yeo, T., Genon, S. in press. Imaging-based parcellations of the human brain. Nature Review Neuroscience.

Evans AC. 2013. Networks of anatomical covariance. NeuroImage. 80:489-504.

Fanselow MS, Dong HW. 2010. Are the dorsal and ventral hippocampus functionally distinct structures? Neuron. 65(1):7-19.

Genon S, Li H, Fan L, Muller VI, Cieslik EC, Hoffstaedter F, Reid AT, Langner R, Grefkes C, Fox PT et al. 2017. The right dorsal premotor mosaic: Organization, functions, and connectivity. Cerebral cortex (New York, NY : 1991). 27(3):2095-2110.

Genon S, Reid A, Langner R, Amunts K, Eickhoff SB. 2018a. How to characterize the function of a brain region. Trends in cognitive sciences.

Genon S, Reid A, Langner R, Amunts K, Eickhoff SB. 2018b. How to characterize the function of a brain region. Trends in cognitive sciences. 22(4):350-364.

Genon S, Reid A, Li H, Fan L, Muller VI, Cieslik EC, Hoffstaedter F, Langner R, Grefkes C, Laird AR et al. 2018c. The heterogeneity of the left dorsal premotor cortex evidenced by multimodal connectivity-based parcellation and functional characterization. NeuroImage. 170:400-411.

Glasser MF, Coalson TS, Robinson EC, Hacker CD, Harwell J, Yacoub E, Ugurbil K, Andersson J, Beckmann CF, Jenkinson M et al. 2016. A multi-modal parcellation of human cerebral cortex. Nature. 536(7615):171-178.

Griffanti L, Salimi-Khorshidi G, Beckmann CF, Auerbach EJ, Douaud G, Sexton CE, Zsoldos E, Ebmeier KP, Filippini N, Mackay CE et al. 2014. Ica-based artefact removal and accelerated fmri acquisition for improved resting state network imaging. NeuroImage. 95:232-247.

Guzowski JF, Knierim JJ, Moser EI. 2004. Ensemble dynamics of hippocampal regions ca3 and ca1. Neuron. 44(4):581-584.

Hallquist MN, Hwang K, Luna B. 2013. The nuisance of nuisance regression: Spectral misspecification in a common approach to resting-state fmri preprocessing reintroduces noise and obscures functional connectivity. NeuroImage. 82:208225.

Hutchison RM, Womelsdorf T, Gati JS, Everling S, Menon RS. 2013. Resting-state networks show dynamic functional connectivity in awake humans and anesthetized macaques. Human brain mapping. 34(9):2154-2177.

Jo HJ, Gotts SJ, Reynolds RC, Bandettini PA, Martin A, Cox RW, Saad ZS. 2013. Effective preprocessing procedures virtually eliminate distance-dependent motion artifacts in resting state fmri. Journal of applied mathematics. 2013. 
Jo HJ, Saad ZS, Simmons WK, Milbury LA, Cox RW. 2010. Mapping sources of correlation in resting state fmri, with artifact detection and removal. NeuroImage. 52(2):571582.

Johansen-Berg H, Behrens TE, Robson MD, Drobnjak I, Rushworth MF, Brady JM, Smith SM, Higham DJ, Matthews PM. 2004. Changes in connectivity profiles define functionally distinct regions in human medial frontal cortex. Proceedings of the National Academy of Sciences of the United States of America. 101(36):1333513340.

Jung M, Wiener S, McNaughton B. 1994. Comparison of spatial firing characteristics of units in dorsal and ventral hippocampus of the rat. The Journal of Neuroscience. 14(12):7347-7356.

Kelly C, Toro R, Di Martino A, Cox CL, Bellec P, Castellanos FX, Milham MP. 2012. A convergent functional architecture of the insula emerges across imaging modalities. NeuroImage. 61(4):1129-1142.

Kim H. 2015. Encoding and retrieval along the long axis of the hippocampus and their relationships with dorsal attention and default mode networks: The hernet model. Hippocampus. 25(4):500-510.

Koster R, Chadwick MJ, Chen Y, Berron D, Banino A, Duzel E, Hassabis D, Kumaran D. 2018. Big-loop recurrence within the hippocampal system supports integration of information across episodes. Neuron. 99(6):1342-1354.e1346.

Kotkowski E, Price LR, Mickle Fox P, Vanasse TJ, Fox PT. 2018. The hippocampal network model: A transdiagnostic metaconnectomic approach. NeuroImage Clinical. 18:115-129.

LaConte S, Anderson J, Muley S, Ashe J, Frutiger S, Rehm K, Hansen LK, Yacoub E, Hu X, Rottenberg D et al. 2003. The evaluation of preprocessing choices in singlesubject bold fmri using npairs performance metrics. NeuroImage. 18(1):10-27.

Laird AR, Eickhoff SB, Fox PM, Uecker AM, Ray KL, Saenz JJ, Jr., McKay DR, Bzdok D, Laird RW, Robinson JL et al. 2011. The brainmap strategy for standardization, sharing, and meta-analysis of neuroimaging data. BMC research notes. 4:349.

Lambert C, Simon H, Colman J, Barrick TR. 2017. Defining thalamic nuclei and topographic connectivity gradients in vivo. NeuroImage. 158:466-479.

Lepage M, Habib R, Tulving E. 1998. Hippocampal pet activations of memory encoding and retrieval: The hiper model. Hippocampus. 8(4):313-322.

Liu TT. 2016. Noise contributions to the fmri signal: An overview. NeuroImage. 143:141151.

Macey PM, Macey KE, Kumar R, Harper RM. 2004. A method for removal of global effects from fmri time series. NeuroImage. 22(1):360-366.

Mechelli A, Friston KJ, Frackowiak RS, Price CJ. 2005. Structural covariance in the human cortex. The Journal of neuroscience : the official journal of the Society for Neuroscience. 25(36):8303-8310.

Meilă M. 2007. Comparing clusterings-an information based distance. Journal of Multivariate Analysis. 98(5):873-895.

Morris RG, Hagan JJ, Rawlins JN. 1986. Allocentric spatial learning by hippocampectomised rats: A further test of the "spatial mapping" and "working memory" theories of hippocampal function. The Quarterly journal of experimental psychology B, Comparative and physiological psychology. 38(4):365-395.

Moser E, Moser MB, Andersen P. 1993. Spatial learning impairment parallels the magnitude of dorsal hippocampal lesions, but is hardly present following ventral 
lesions. The Journal of neuroscience : the official journal of the Society for Neuroscience. 13(9):3916-3925.

Moser MB, Moser EI. 1998. Functional differentiation in the hippocampus. Hippocampus. 8(6):608-619.

Mueller S, Wang D, Fox MD, Yeo BT, Sepulcre J, Sabuncu MR, Shafee R, Lu J, Liu H. 2013. Individual variability in functional connectivity architecture of the human brain. Neuron. 77(3):586-595.

Murphy K, Birn RM, Handwerker DA, Jones TB, Bandettini PA. 2009. The impact of global signal regression on resting state correlations: Are anti-correlated networks introduced? NeuroImage. 44(3):893-905.

Nanetti L, Cerliani L, Gazzola V, Renken R, Keysers C. 2009. Group analyses of connectivity-based cortical parcellation using repeated k-means clustering. NeuroImage. 47(4):1666-1677.

Ojemann JG, Akbudak E, Snyder AZ, McKinstry RC, Raichle ME, Conturo TE. 1997. Anatomic localization and quantitative analysis of gradient refocused echoplanar fmri susceptibility artifacts. NeuroImage. 6(3):156-167.

Oppenheim C, Dormont D, Biondi A, Lehericy S, Hasboun D, Clemenceau S, Baulac M, Marsault C. 1998. Loss of digitations of the hippocampal head on high-resolution fast spin-echo mr: A sign of mesial temporal sclerosis. AJNR American journal of neuroradiology. 19(3):457-463.

Petrovich GD, Canteras NS, Swanson LW. 2001. Combinatorial amygdalar inputs to hippocampal domains and hypothalamic behavior systems. Brain research Brain research reviews. 38(1-2):247-289.

Poppenk J, Evensmoen HR, Moscovitch M, Nadel L. 2013. Long-axis specialization of the human hippocampus. Trends in cognitive sciences. 17(5):230-240.

Power JD, Barnes KA, Snyder AZ, Schlaggar BL, Petersen SE. 2012. Spurious but systematic correlations in functional connectivity mri networks arise from subject motion. NeuroImage. 59(3):2142-2154.

Power JD, Mitra A, Laumann TO, Snyder AZ, Schlaggar BL, Petersen SE. 2014. Methods to detect, characterize, and remove motion artifact in resting state fmri. NeuroImage. 84:320-341.

Power JD, Schlaggar BL, Petersen SE. 2015. Recent progress and outstanding issues in motion correction in resting state fmri. NeuroImage. 105:536-551.

Prince SE, Daselaar SM, Cabeza R. 2005. Neural correlates of relational memory: Successful encoding and retrieval of semantic and perceptual associations. The Journal of neuroscience : the official journal of the Society for Neuroscience. 25(5):1203-1210.

Reid AT, Bzdok D, Langner R, Fox PT, Laird AR, Amunts K, Eickhoff SB, Eickhoff CR. 2016. Multimodal connectivity mapping of the human left anterior and posterior lateral prefrontal cortex. Brain structure \& function. 221(5):2589-2605.

Risold PY, Swanson LW. 1996. Structural evidence for functional domains in the rat hippocampus. Science (New York, NY). 272(5267):1484-1486.

Robinson JL, Barron DS, Kirby LA, Bottenhorn KL, Hill AC, Murphy JE, Katz JS, Salibi N, Eickhoff SB, Fox PT. 2015. Neurofunctional topography of the human hippocampus. Human brain mapping. 36(12):5018-5037.

Robinson JL, Salibi N, Deshpande G. 2016. Functional connectivity of the left and right hippocampi: Evidence for functional lateralization along the long-axis using meta-analytic approaches and ultra-high field functional neuroimaging. NeuroImage. 135:64-78. 
Salimi-Khorshidi G, Douaud G, Beckmann CF, Glasser MF, Griffanti L, Smith SM. 2014. Automatic denoising of functional mri data: Combining independent component analysis and hierarchical fusion of classifiers. Neurolmage. 90:449-468.

Satterthwaite TD, Ciric R, Roalf DR, Davatzikos C, Bassett DS, Wolf DH. 2017. Motion artifact in studies of functional connectivity: Characteristics and mitigation strategies. Human brain mapping.

Satterthwaite TD, Elliott MA, Gerraty RT, Ruparel K, Loughead J, Calkins ME, Eickhoff SB, Hakonarson H, Gur RC, Gur RE et al. 2013. An improved framework for confound regression and filtering for control of motion artifact in the preprocessing of resting-state functional connectivity data. NeuroImage. 64:240-256.

Schaefer A, Kong R, Gordon EM, Laumann TO, Zuo XN, Holmes AJ, Eickhoff SB, Yeo BTT. 2017. Local-global parcellation of the human cerebral cortex from intrinsic functional connectivity mri. Cerebral cortex (New York, NY : 1991).1-20.

Seeley WW, Crawford RK, Zhou J, Miller BL, Greicius MD. 2009. Neurodegenerative diseases target large-scale human brain networks. Neuron. 62(1):42-52.

Small SA, Nava AS, Perera GM, DeLaPaz R, Mayeux R, Stern Y. 2001. Circuit mechanisms underlying memory encoding and retrieval in the long axis of the hippocampal formation. Nature neuroscience. 4(4):442-449.

Smith SM, Beckmann CF, Andersson J, Auerbach EJ, Bijsterbosch J, Douaud G, Duff E, Feinberg DA, Griffanti L, Harms MP et al. 2013. Resting-state fmri in the human connectome project. NeuroImage. 80:144-168.

Strange BA, Fletcher PC, Henson RN, Friston KJ, Dolan RJ. 1999. Segregating the functions of human hippocampus. Proceedings of the National Academy of Sciences of the United States of America. 96(7):4034-4039.

Strange BA, Witter MP, Lein ES, Moser EI. 2014. Functional organization of the hippocampal longitudinal axis. Nature reviews Neuroscience. 15(10):655-669.

Strother SC, Anderson J, Hansen LK, Kjems U, Kustra R, Sidtis J, Frutiger S, Muley S, LaConte S, Rottenberg D. 2002. The quantitative evaluation of functional neuroimaging experiments: The npairs data analysis framework. NeuroImage. 15(4):747-771.

Swanson LW, Cowan WM. 1977. An autoradiographic study of the organization of the efferent connections of the hippocampal formation in the rat. The Journal of comparative neurology. 172(1):49-84.

Treit S, Steve T, Gross DW, Beaulieu C. 2018. High resolution in-vivo diffusion imaging of the human hippocampus. NeuroImage. 182:479-487.

Ugurbil K, Xu J, Auerbach EJ, Moeller S, Vu AT, Duarte-Carvajalino JM, Lenglet C, Wu X, Schmitter S, Van de Moortele PF et al. 2013. Pushing spatial and temporal resolution for functional and diffusion mri in the human connectome project. NeuroImage. 80:80-104.

Van Dijk KR, Hedden T, Venkataraman A, Evans KC, Lazar SW, Buckner RL. 2010. Intrinsic functional connectivity as a tool for human connectomics: Theory, properties, and optimization. J Neurophysiol. 103(1):297-321.

Van Dijk KR, Sabuncu MR, Buckner RL. 2012. The influence of head motion on intrinsic functional connectivity mri. NeuroImage. 59(1):431-438.

Van Essen DC, Ugurbil K. 2012. The future of the human connectome. NeuroImage. 62(2):1299-1310.

Vann SD, Brown MW, Erichsen JT, Aggleton JP. 2000. Fos imaging reveals differential patterns of hippocampal and parahippocampal subfield activation in rats in response to different spatial memory tests. The Journal of neuroscience : the official journal of the Society for Neuroscience. 20(7):2711-2718. 
Varikuti DP, Genon S, Sotiras A, Schwender H, Hoffstaedter F, Patil KR, Jockwitz C, Caspers S, Moebus S, Amunts K et al. 2018. Evaluation of non-negative matrix factorization of grey matter in age prediction. NeuroImage. 173:394-410.

Varikuti DP, Hoffstaedter F, Genon S, Schwender H, Reid AT, Eickhoff SB. 2017. Restingstate test-retest reliability of a priori defined canonical networks over different preprocessing steps. Brain structure \& function. 222(3):1447-1468.

Weissenbacher A, Kasess C, Gerstl F, Lanzenberger R, Moser E, Windischberger C. 2009. Correlations and anticorrelations in resting-state functional connectivity mri: A quantitative comparison of preprocessing strategies. NeuroImage. 47(4):14081416.

Windischberger C, Langenberger H, Sycha T, Tschernko EM, Fuchsjager-Mayerl G, Schmetterer L, Moser E. 2002. On the origin of respiratory artifacts in bold-epi of the human brain. Magnetic resonance imaging. 20(8):575-582.

Wise RG, Ide K, Poulin MJ, Tracey I. 2004. Resting fluctuations in arterial carbon dioxide induce significant low frequency variations in bold signal. NeuroImage. 21(4):1652-1664.

Wisse LE, Gerritsen L, Zwanenburg JJ, Kuijf HJ, Luijten PR, Biessels GJ, Geerlings MI. 2012. Subfields of the hippocampal formation at $7 \mathrm{t}$ mri: In vivo volumetric assessment. NeuroImage. 61(4):1043-1049.

Yan CG, Cheung B, Kelly C, Colcombe S, Craddock RC, Di Martino A, Li Q, Zuo XN, Castellanos FX, Milham MP. 2013. A comprehensive assessment of regional variation in the impact of head micromovements on functional connectomics. NeuroImage. 76:183-201.

Yarkoni T, Poldrack RA, Nichols TE, Van Essen DC, Wager TD. 2011. Large-scale automated synthesis of human functional neuroimaging data. Nature methods. 8(8):665-670.

Yee Y, Fernandes DJ, French L, Ellegood J, Cahill LS, Vousden DA, Spencer Noakes L, Scholz J, van Eede MC, Nieman BJ et al. 2017. Structural covariance of brain region volumes is associated with both structural connectivity and transcriptomic similarity. bioRxiv.

Zeidman P, Maguire EA. 2016. Anterior hippocampus: The anatomy of perception, imagination and episodic memory. Nature reviews Neuroscience. 17(3):173-182.

Zhang X, Mormino EC, Sun N, Sperling RA, Sabuncu MR, Yeo BT. 2016. Bayesian model reveals latent atrophy factors with dissociable cognitive trajectories in alzheimer's disease. Proceedings of the National Academy of Sciences of the United States of America. 113(42):E6535-e6544. 